\title{
Thermodynamic formalism, topological pressure, and escape rates for critically non-recurrent conformal dynamics
}

\author{
by
}

Mariusz Urbański (Denton, TX)

\begin{abstract}
We show that for critically non-recurrent rational functions all the definitions of topological pressure proposed in [12] coincide for all $t \geq 0$. Then we study in detail the Gibbs states corresponding to the potentials $-t \log \left|f^{\prime}\right|$ and their $\sigma$-finite invariant versions. In particular we provide a sufficient condition for their finiteness. We determine the escape rates of critically non-recurrent rational functions. In the presence of parabolic points we also establish a polynomial rate of appropriately modified escape. This extends the corresponding result from [6] proven in the context of parabolic rational functions. In the last part of the paper we introduce the class of critically tame generalized polynomial-like mappings. We show that if $f$ is a critically tame and critically non-recurrent generalized polynomial-like mapping and $g$ is a Hölder continuous potential (with sufficiently large exponent if $f$ has parabolic points) and the topological pressure satisfies $\mathrm{P}(g)>\sup (g)$, then for sufficiently small $\delta>0$, the function $t \mapsto \mathrm{P}(t g)$, $t \in(1-\delta, 1+\delta)$, is real-analytic.
\end{abstract}

1. Introduction and preliminaries. A rational function $f: \overline{\mathbb{C}} \rightarrow \overline{\mathbb{C}}$ is called critically non-recurrent if no critical point contained in its Julia set is recurrent. In [17] and [18] we explored some geometrical and dynamical properties of critically non-recurrent rational functions. In this paper we continue the investigations originated in these two papers. More precisely, in Section 2 we deal with various generalizations of topological pressure $\mathrm{P}(t)$ of the potential $-t \log \left|f^{\prime}\right|, t \geq 0$, proposed in [12]. We demonstrate (see Theorem 2.6) that for critically non-recurrent rational functions all these definitions of topological pressure coincide for all $t \geq 0$. In Section 3 we deal with thermodynamic formalism of critically non-recurrent dynamics. We study in detail the Gibbs states corresponding to the potentials $-t \log \left|f^{\prime}\right|$ and their $\sigma$-finite invariant versions. In particular we provide a sufficient condition for these invariant measures to be finite. In Section 4 we deal with

2000 Mathematics Subject Classification: 37F35, 37F10, 37D35, 37D45.

The research was supported in part by the NSF Grant DMS 0100078. 
escape rates. We show that in the critically non-recurrent case this rate is equal to $\mathrm{P}(2)$. In the presence of parabolic points we establish a polynomial rate of appropriately modified escape. This extends the corresponding result from [6] proven in the context of parabolic rational functions. Our approach differs from Haydn's and Isola's in that we estimate moduli of appropriate annuli and we use McMullen's result relating such moduli to hyperbolic diameters of the corresponding sets enclosed by these annuli. In Section 5, we deal with generalized polynomial-like maps and Hölder continuous potentials. We show that if $f$ is a critically tame and critically non-recurrent generalized polynomial-like mapping and $g$ is a Hölder continuous potential (with sufficiently large exponent if $f$ has parabolic points) and the topological pressure satisfies $\mathrm{P}(g)>\sup (g)$, then for sufficiently small $\delta>0$, the function $t \mapsto \mathrm{P}(t g), t \in(1-\delta, 1+\delta)$, is real-analytic.

2. Various pressures. Let $f: \overline{\mathbb{C}} \rightarrow \overline{\mathbb{C}}$ be a rational function on the Riemann sphere $\overline{\mathbb{C}}$ of degree $\geq 2$. In [12] F. Przytycki proposed several ways of extending the concept of topological pressure of the potential $-t \log \left|f^{\prime}\right|$, $t \geq 0$, to the general case. Let us describe them briefly:

1. Variational pressure:

$$
\mathrm{P}_{\mathrm{var}}(t)=\sup \left\{h_{\mu}(f)-t \int \log \left|f^{\prime}\right| d \mu\right\},
$$

where the supremum is taken over all ergodic $f$-invariant measures on $J(f)$.

2. Hyperbolic variational pressure:

$$
\mathrm{P}_{\text {hypvar }}(t)=\sup \left\{h_{\mu}(f)-t \int \log \left|f^{\prime}\right| d \mu\right\},
$$

where the supremum is taken over all ergodic $f$-invariant measures on $J(f)$ with positive Lyapunov exponent, i.e. such that $\chi_{\mu}(f)=\int \log \left|f^{\prime}\right| d \mu>0$.

3. Hyperbolic pressure. We call a forward invariant compact set $X \subset J(f)$ hyperbolic if there exists $n \geq 1$ such that for every $x \in X,\left|\left(f^{n}\right)^{\prime}(x)\right|>1$. The hyperbolic pressure is

$$
\mathrm{P}_{\text {hyp }}(t)=\sup _{X}\left\{\mathrm{P}\left(\left.f\right|_{X},-t \log \left|f^{\prime}\right|\right)\right\},
$$

where the supremum is taken over all $f$-invariant hyperbolic subsets $X$ of $J(f)$ such that an iterate of $\left.f\right|_{X}$ is topologically conjugate to a subshift of finite type.

4. DU pressure. Let $V$ be an open subset of $J(f)$ such that $J(f) \cap$ $\operatorname{Crit}(f) \subset V$ and let

$$
K(V)=J(f) \backslash \bigcup_{n \geq 0} f^{-n}(V)
$$

Since $K(V)$ is compact, $f$-invariant and disjoint from $\operatorname{Crit}(f)$, we can con- 
sider the standard topological pressure $\mathrm{P}\left(\left.f\right|_{K(V)},-t \int \log \left|f^{\prime}\right|\right)$. Put

$$
\mathrm{P}_{\mathrm{DU}}(t)=\sup _{V}\left\{\mathrm{P}\left(\left.f\right|_{K(V)},-t \log \left|f^{\prime}\right|\right)\right\}
$$

where the supremum is taken over all open sets $V$ considered above.

5. Minimal conformal eigenvalue. The minimal conformal eigenvalue $\lambda(t)$ is defined to be the infimum of all $\lambda>0$ for which there exists a Borel probability measure $m$ such that

$$
\frac{d(m \circ f)}{d m}=\lambda\left|f^{\prime}\right|^{t}
$$

We set

$$
\mathrm{P}_{\mathrm{c}}(t)=\log \lambda(t)
$$

6. Point pressure. Given $z \in \overline{\mathbb{C}} \backslash \bigcup_{n \geq 0} f^{n}(\operatorname{Crit}(f))$ and $t \geq 0$ put

$$
\mathrm{P}_{z}(t)=\limsup _{n \rightarrow \infty} \frac{1}{n} \sum_{x \in f^{-n}(z)}\left|\left(f^{n}\right)^{\prime}(x)\right|^{-t} .
$$

F. Przytycki proved in [12] that there exists a set $G \subset \overline{\mathbb{C}} \backslash \bigcup_{n \geq 0} f^{n}(\operatorname{Crit}(f))$ such that $\operatorname{HD}(\overline{\mathbb{C}} \backslash G)=0$ and $\mathrm{P}_{z}(t)=\mathrm{P}_{w}(t)$ for all $z, w \in G$. This common value will be denoted by $\mathrm{P}_{\mathrm{p}}(t)$. It is not difficult to check (see [12]) that the following proposition is true.

Proposition 2.1. All the pressures defined in items 1-6 are Lipschitz continuous and monotone with respect to the variable $t$.

The following fact has been proved in [12] (cf. [4] and [14]).

THEOREM 2.2. There exists a number $h=h(f)$ called the Poincaré exponent of the function $f$ in [12] and called the dynamical dimension of the Julia set in [4] such that all the pressures defined in items 1-6 coincide on the interval $[0, h]$, are positive on $[0, h)$ and vanish at $h$.

Our aim in this section is to extend this equality of pressures to the whole set $[0, \infty)$ in the case of critically non-recurrent dynamics. We start with the following.

LEMMA 2.3. If $f$ is critically non-recurrent and $y \in J(f)$ is a periodic point of $f$, say of period $q$, then there exists a sequence $\left\{y_{n}\right\}_{n=1}^{\infty} \subset J(f)$ of periodic points of $f$, all different from $y$, of respective periods $q_{n}$, such that $\lim _{n \rightarrow \infty} y_{n}=y$ and

$$
\lim _{n \rightarrow \infty} \frac{1}{q_{n}} \log \left|\left(f^{q_{n}}\right)^{\prime}\left(y_{n}\right)\right| \leq \frac{1}{q} \log \left|\left(f^{q}\right)^{\prime}(y)\right| .
$$

In addition, if there exists no $k \geq 1$ such that $f^{-k}(y) \backslash\left\{f^{j}(y)\right.$ : $0 \leq j \leq q-1\} \subset \operatorname{Crit}(f)$ or if $y \in \Omega$, then this inequality can be replaced by equality. 
Proof. Our strategy is to approximate $y$ by periodic points of $f^{q}$. Without loss of generality we may assume that $q=1$. Regardless of whether $y$ is repelling or rationally indifferent there exist $\theta>0, x \in J(f) \cap B(y, \theta)$ and $\varepsilon>0$ such that all the local holomorphic inverse branches $f_{y}^{-n}$ of all iterates of $f$ are defined on $\bar{B}(x, 2 \varepsilon) \subset B(y, \theta) \backslash\{y\}$, the closed ball centered at $x$ and with radius $2 \varepsilon$. Local means here that $\lim _{n \rightarrow \infty} f_{y}^{-n}(x)=y$ and $f_{y}^{-n}(\bar{B}(x, 2 \varepsilon)) \subset B(y, \theta)$. In the case when $y$ is a repelling point all these branches are defined on the entire ball $B(y, \theta)$ for $\theta>0$ small enough. Since $\overline{\bigcup_{n \geq 0} f^{n}(\operatorname{Crit}(f))}$ is, by Lemma 5.2 of [17], nowhere dense in $\left.J(f)\right)$, there exists a closed ball $B \subset 2 B \subset B(x, \varepsilon)$ centered at a point $w \in J(f)$ such that $2 B \cap \overline{\bigcup_{n \geq 0} f^{n}(\operatorname{Crit}(f))}=\emptyset$. This implies that $f_{y}^{-n}(2 B) \cap \overline{\bigcup_{n \geq 0} f^{n}(\operatorname{Crit}(f))}$ $=\emptyset$ for every $n \geq 0$, and since $f: J(f) \rightarrow J(f)$ is topologically exact, there exists $l \geq 1$ independent of $n$ and a holomorphic branch $f_{*}^{-l}: f_{y}^{-n}(2 B) \rightarrow \overline{\mathbb{C}}$ of $f^{-l}$ sending $f_{y}^{-n}(w)$ to $B$. Therefore, for every $n \geq 1$ large enough, $f_{*}^{-l} \circ f_{y}^{-n}(2 B) \subset 2 B$. Hence by the Brouwer fixed point theorem there exists a fixed point $y_{n+l} \in 2 B$ of $f_{*}^{-l} \circ f_{y}^{-n}: 2 B \rightarrow 2 B$. Hence $f^{n+l}\left(y_{n+l}\right)=y_{n+l}$ and $y_{n+l} \neq y$ as, by the choice of $\varepsilon, y \notin 2 B$. It is clear that in the repelling case

$$
\lim _{n \rightarrow \infty} \frac{1}{n} \log \left|\left(f^{n}\right)^{\prime}\left(y_{n}\right)\right| \leq \log \left|f^{\prime}(y)\right|
$$

in the parabolic case this follows from the fact that $\left|\left(f_{y}^{-n}\right)^{\prime}(w)\right| \asymp n^{-(p+1) / p}$ for all $w \in B(x, \varepsilon)$, where $p \geq 1$ is the number of petals of the point $y$. If there exists no $k \geq 1$ such that $f^{-k}(y) \backslash\left\{f^{j}(y): 0 \leq j \leq q-1\right\} \subset \operatorname{Crit}(f)$, then $f_{*}^{-l}$ extends holomorphically onto $B(y, \kappa)$ for some $\kappa>0$ sufficiently small and the inequality (2.1) becomes an equality. If $y \in \Omega$ and $f^{-k}(y) \backslash\left\{f^{j}(y): 0 \leq\right.$ $j \leq q-1\} \subset \operatorname{Crit}(f)$, then $\left|\left(f_{y}^{-n}\right)^{\prime}\left(y_{n+l}\right)\right| \asymp n^{-(p+1) / p}$ and $\left|f_{y}^{-n}\left(y_{n+l}\right)-y\right| \asymp$ $n^{-1 / p}$. If $l<k$, we conclude the proof as above. If $k \leq l$, passing to a subsequence, we may assume that $\lim _{n \rightarrow \infty} y_{n+l}=c$, a critical point of $f^{l}$ belonging to $f^{-l}(y)$. Denote the order of the critical point $c$ of $f^{l}$ by $s$. We then obtain

$$
\begin{aligned}
\left|\left(f_{*}^{-l} \circ f_{y}^{-n}\right)^{\prime}\left(y_{n+l}\right)\right| & =\left|\left(f_{*}^{-l}\right)^{\prime}\left(f_{y}^{-n}\left(y_{n+l}\right)\right)\right| \cdot\left|\left(f_{y}^{-n}\right)^{\prime}\left(y_{n+l}\right)\right| \\
& \asymp\left(n^{-1 / p}\right)^{1 / s-1} \cdot n^{-(p+1) / p}=n^{-(1+1 /(s p))} .
\end{aligned}
$$

Thus $\left|\left(f^{n+l}\right)^{\prime}\left(y_{n+l}\right)\right| \asymp n^{1+1 /(s p)}$ and therefore

$$
\lim _{n \rightarrow \infty} \frac{1}{n+l} \log \left|\left(f^{n+l}\right)^{\prime}\left(y_{n+l}\right)\right|=0
$$

Let $\Omega$ be the set of all parabolic points of $f$, i.e.

$$
\Omega=\left\{\omega \in J(f): \exists_{q \geq 1} f^{q}(\omega)=\omega \text { and }\left(f^{q}\right)^{\prime}(\omega)=1\right\} .
$$

Lemma 2.4. Assume that $f$ is critically non-recurrent. If $\mu$ is a Borel probability $f$-invariant ergodic measure supported on $J(f)$, then either

$$
\chi_{\mu}=\int \log \left|f^{\prime}\right| d \mu>0 \quad \text { or } \quad \mu(\Omega)=1 .
$$


Proof. Suppose that $\mu(\Omega)<1$. Since $\Omega$ consists of periodic points and since $\mu$ is ergodic, this implies that $\mu(\Omega)=0$. Since no critical point contained in the Julia set of $f$ is periodic, we conclude that

$$
\mu\left(\bigcup_{n \geq 0} f^{-n}(\Omega \cup(\operatorname{Crit}(f) \cap J(f)))\right)=0 .
$$

Hence, by Birkhoff's ergodic theorem there exists $z \in J(f) \backslash \bigcup_{n \geq 0} f^{-n}(\Omega \cup$ $(\operatorname{Crit}(f) \cap J(f)))$ such that

$$
\int \log \left|f^{\prime}\right| d \mu=\lim _{n \rightarrow \infty} \frac{1}{n} \log \left|\left(f^{n}\right)^{\prime}(z)\right| .
$$

In view of Proposition 6.1 from [17] there exist a sequence $\left\{n_{j}\right\}_{j=1}^{\infty}$ (depending on $z$ ) increasing to infinity and a number $\eta(z)>0$ such that

$$
C_{n_{j}}\left(z, B\left(f^{n_{j}}(z), \eta(z)\right)\right) \cap \operatorname{Crit}\left(f^{n_{j}}\right)=\emptyset,
$$

where $C_{n}(z, F)$ is the connected component of $f^{-n}(F)$ containing $z$. We may assume that $\eta(z) \leq \eta$, where $\eta>0$ is the constant appearing in Lemma 7.7 of [18]. It therefore follows from Koebe's distortion theorem that

$$
\left|\left(f^{n_{j}}\right)^{\prime}(z)\right| \asymp \operatorname{diam}\left(C_{n_{j}}\left(z, B\left(f^{n_{j}}(z), \eta(z) / 2\right)\right)\right) .
$$

Choose $\theta>0$ used in the definition of the operation Comp $p_{*}^{k}$ from [18]. Then for every $n \geq 1$ there exists a unique $n^{*} \leq n$ such that $z \in$ $\mathrm{Comp}_{*}^{n^{*}}\left(B\left(f^{n_{j}}(z), \eta(z) / 2\right)\right)=C_{n}\left(z, B\left(f^{n}(z), \eta(z)\right)\right)$. Combining (2.3) and Lemma 7.7 of [18] we obtain

$$
\liminf _{j \rightarrow \infty} \frac{1}{n_{j}^{*}} \log \left|\left(f^{n_{j}}\right)^{\prime}(z)\right|>0 .
$$

Since $\mu(\Omega)=0$, we may require $\theta>0$ to be so small that $\mu(B(\Omega, 2 \theta))<1 / 2$. Applying now Birkhoff's ergodic theorem we deduce that we could choose $z$ to satisfy

$$
\liminf _{j \rightarrow \infty} \frac{n_{j}^{*}}{n_{j}} \geq \lim _{n \rightarrow \infty} \frac{1}{n} \sum_{j=0}^{n-1} \mathbb{1}_{J(f) \backslash B(\Omega, 2 \theta)} \circ f^{j}(z)>1 / 2 .
$$

Combining this, (2.4) and (2.2), we conclude that $\int \log \left|f^{\prime}\right| d \mu>0$.

As an immediate consequence of this lemma we get the following.

COROLlary 2.5. If $f$ is semi-hyperbolic (critically non-recurrent and $\Omega=\emptyset)$, then $\int \log \left|f^{\prime}\right| d \mu>0$ for every Borel probability $f$-invariant ergodic measure $\mu$ supported on $J(f)$.

The main result of this section is the following.

Theorem 2.6. Assume that $f$ is critically non-recurrent. Then

(a) All the pressures defined in items 1-6 coincide throughout the whole interval $[0, \infty)$; denote their common value by $\mathrm{P}(t)$.

(b) If $\Omega=\emptyset$, then $\mathrm{P}(t)<0$ for all $t>h$. 
(c) If $\Omega \neq \emptyset$, then $\mathrm{P}(t)=0$ for all $t \geq h$.

(d) $h=\mathrm{HD}(J(f))$, the Hausdorff dimension of the Julia set $J(f)$.

Proof. Item (d) is an immediate consequence of the results obtained in [17]. It follows from the facts established in the course of the proof of Theorem A2.9 in [12] that

$$
\mathrm{P}_{\mathrm{c}}(t)=\mathrm{P}_{\mathrm{p}}(t) \geq \mathrm{P}_{\text {hypvar }}(t)=\mathrm{P}_{\text {hyp }}(t) \leq \mathrm{P}_{\mathrm{var}}(t) \geq \mathrm{P}_{\mathrm{DU}}(t) .
$$

Thus, in order to complete the proof of item (a), it suffices to show that

$$
\mathrm{P}_{\mathrm{var}}(t)=\mathrm{P}_{\text {hypvar }}(t)
$$

and

$$
\mathrm{P}_{\mathrm{DU}}(t) \geq \mathrm{P}_{\mathrm{c}}(t) \text {. }
$$

Indeed, if $\Omega=\emptyset$, then (2.5) follows immediately from Lemma 2.4. If $\Omega \neq \emptyset$ and $t>h$, then in view of Proposition 2.1, $\mathrm{P}_{\mathrm{var}}(t)=0$. Using in addition Lemma 2.4, we see that also $\mathrm{P}_{\text {hypvar }}(t)=0$. Therefore, applying Theorem 2.2, we conclude the proof of formula (2.5).

In order to prove (2.6) we shall construct a Borel probability measure $m$ on $J(f)$ such that $d(m \circ f) / d m=e^{\widehat{\mathrm{P}}(t)}\left|f^{\prime}\right|^{t}$ for some $\widehat{\mathrm{P}}(t) \leq \mathrm{P}_{\mathrm{DU}}(t)$. Indeed, for every $c \in \operatorname{Crit}(f) \cap J(f)$ there exists $y_{c} \in \omega(c) \backslash \bigcup_{n \geq 0} f^{-n}(\operatorname{Crit}(f) \cap J(f))$. For every $n \geq 1$ let

$$
V_{n}=\bigcup_{c \in \operatorname{Crit}(f) \cap J(f)} B\left(y_{c}, 1 / n\right) .
$$

Then for all $n$ large enough, $V_{n} \cap \operatorname{Crit}(f)=\emptyset$. In addition, for every $c \in$ $\operatorname{Crit}(f) \cap J(f)$ there exists $k(c) \geq 1$ such that $f^{k(c)}(c) \in V_{n}$ and

$$
K\left(V_{n}\right) \subset K\left(\bigcup_{c \in \operatorname{Crit}(f) \cap J(f)} f^{-k(c)}\left(V_{n}\right)\right) .
$$

Thus

$$
\begin{aligned}
\mathrm{P}_{n}(t) & :=\mathrm{P}\left(\left.f\right|_{K\left(V_{n}\right)},-t \log \left|f^{\prime}\right|\right) \\
& \leq \mathrm{P}\left(\left.f\right|_{K\left(\bigcup_{c \in \operatorname{Crit}(f) \cap J(f)} f^{-k(c)}\left(V_{n}\right)\right)},-t \log \left|f^{\prime}\right|\right) \leq \mathrm{P}_{\mathrm{DU}}(t) .
\end{aligned}
$$

Since it is not difficult to see that $\left.f\right|_{K\left(\bigcup_{c \in \operatorname{Crit}(f) \cap J(f)} f^{\left.-k(c)\left(V_{n}\right)\right)}\right.}$ is expansive and consequently so is $\left.f\right|_{K\left(V_{n}\right)}$, it follows from Theorem 3.12 of [2] that there exists a Borel probability measure $m_{n}$ supported on $K\left(V_{n}\right)$ for which

$$
m_{n}(f(A))=\int_{A} e^{\mathrm{P}_{n}(t)}\left|f^{\prime}\right|^{t} d m_{n}
$$

for every Borel set $A \subset K\left(V_{n}\right) \backslash \partial V_{n}$ ( $\partial V_{n}$ is the only set where the map 
$\left.f\right|_{K\left(V_{n}\right)}$ may fail to be open) such that $\left.f\right|_{A}$ is 1-to-1, and

$$
m_{n}(f(A)) \geq \int_{A} e^{\mathrm{P}_{n}(t)}\left|f^{\prime}\right|^{t} d m_{n}
$$

for every Borel set $A \subset K\left(V_{n}\right)$ such that $\left.f\right|_{A}$ is 1-to-1. A straightforward analysis (see [4] for details) shows that (2.8) continues to hold for all sets $A \subset J(f) \backslash V_{n}$ and (2.9) continues to hold for all sets $A \subset J(f)$ satisfying in each case the requirement that $\left.f\right|_{A}$ is 1-to-1. Let $m$ be a weak limit of the measures $m_{n}$ as $n \nearrow \infty$. Since $\mathrm{P}_{n}(t)$ is an increasing function, the limit $\widehat{\mathrm{P}}(t)=\lim _{n \rightarrow \infty} \mathrm{P}_{n}(t)$ exists and by $(2.7), \widehat{\mathrm{P}}(t) \leq \mathrm{P}_{\mathrm{DU}}(t)$. Proceeding as in the proof of Lemma 5.5 in [4] (cf. [14]) we conclude that

$$
m(f(A))=\int_{A} e^{\widehat{\mathrm{P}}(t)}\left|f^{\prime}\right|^{t} d m
$$

for every Borel set $A \subset J(f) \backslash\left\{y_{c}: c \in \operatorname{Crit}(f)\right\}$ such $\left.f\right|_{A}$ is 1-to-1, and

$$
m(f(A)) \geq \int_{A} e^{\widehat{\mathrm{P}}(t)}\left|f^{\prime}\right|^{t} d m
$$

for every Borel set $A \subset J(f)$ such $\left.f\right|_{A}$ is 1-to-1. In order to proceed further we need to impose more restrictions on the choice of points $y_{c}$. Namely, since for every $c \in \operatorname{Crit}(f), \omega(c)$ is compact and $f(\omega(c)) \subset \omega(c)$, there exists a Borel probability $f$-invariant ergodic measure $\mu_{c}$ supported on $\omega(c)$. Fix an arbitrary point $y_{c} \in \operatorname{supp}(\omega(c))$ which is recurrent and such that

$$
\lim _{n \rightarrow \infty} \frac{1}{n} \log \left|\left(f^{n}\right)^{\prime}\left(y_{c}\right)\right|=\chi_{\mu_{c}}=\int \log \left|f^{\prime}\right| d \mu_{c} .
$$

Our aim is to show that (2.10) is also satisfied for the singleton $A=\left\{y_{c}\right\}$. Suppose first that $y_{c}$ is eventually periodic. Since $y_{c}$ is recurrent, it must be periodic. Fix $\varepsilon>0$. In view of Lemma 2.3 there exists a periodic point $x_{c}$ whose periodic orbit is disjoint from $\operatorname{Crit}(f)$ and such that

$$
\frac{1}{q} \log \left|\left(f^{q}\right)^{\prime}\left(x_{c}\right)\right| \leq \chi_{\mu_{c}}+\varepsilon,
$$

where $q \geq 1$ is the shortest period of $x_{c}$. Let $\mu_{q}$ be the atomic probability measure equidistributed on the forward orbit of $x_{c}$. Of course $\mu_{q}$ is ergodic and $f$-invariant. Since $x_{c} \in K\left(V_{n}\right)$ for all $n \geq 1$ large enough, we get

$$
\widehat{\mathrm{P}}(t) \geq \mathrm{P}_{n}(t) \geq \mathrm{h}_{\mu_{q}}-t \log \left|f^{\prime}\right| d \mu_{q}=-t \frac{1}{q} \log \left|\left(f^{q}\right)^{\prime}\left(x_{c}\right)\right| \geq-t \chi_{\mu_{c}}-t \varepsilon .
$$

Letting $\varepsilon \searrow 0$ we therefore get $\widehat{\mathrm{P}}(t) \geq-t \chi_{\mu_{c}}=-(t / p) \log \left|\left(f^{p}\right)^{\prime}\left(y_{c}\right)\right|$, where $p \geq 1$ is the shortest period of periodic point $y_{c}$. Equivalently,

$$
e^{p \widehat{\mathrm{P}}(t)}\left|\left(f^{p}\right)^{\prime}\left(y_{c}\right)\right|^{t} \geq 1 \quad \text { or } \quad e^{p \widehat{\mathrm{P}}(t)}\left|\left(f^{p}\right)^{\prime}\left(f\left(y_{c}\right)\right)\right|^{t} \geq 1 .
$$


Using this and applying (2.11) consecutively to the sets $\left\{f^{p}\left(y_{c}\right)\right\},\left\{f^{p-1}\left(y_{c}\right)\right\}$, $\ldots,\left\{f\left(y_{c}\right)\right\}$, we get

$$
\begin{aligned}
m\left(f\left(y_{c}\right)\right) & =m\left(f\left(f^{p}\left(y_{c}\right)\right)\right) \geq \int_{f^{p}\left(y_{c}\right)} e^{\widehat{\mathrm{P}}(t)}\left|f^{\prime}\right|^{t} d m \\
& \geq \int_{f^{p-1}\left(y_{c}\right)} e^{2 \widehat{\mathrm{P}}(t)}\left|\left(f^{2}\right)^{\prime}\left(f\left(y_{c}\right)\right)\right|^{t} d m \geq \ldots \\
& \geq \int_{f\left(y_{c}\right)} e^{p \widehat{\mathrm{P}}(t)}\left|\left(f^{p}\right)^{\prime}\left(f\left(y_{c}\right)\right)\right|^{t} d m \\
& =e^{p \widehat{\mathrm{P}}(t)}\left|\left(f^{p}\right)^{\prime}\left(f\left(y_{c}\right)\right)\right| m\left(f\left(y_{c}\right)\right) \geq m\left(f\left(y_{c}\right)\right) .
\end{aligned}
$$

So, all the inequalities in this formula are in fact equalities and in particular

$$
m\left(f\left(y_{c}\right)\right)=\int_{f^{p}\left(y_{c}\right)} e^{\widehat{\mathrm{P}}(t)}\left|f^{\prime}\right|^{t} d m=\int_{y_{c}} e^{\widehat{\mathrm{P}}(t)}\left|f^{\prime}\right|^{t} d m,
$$

and we are done in this case.

So, suppose that the point $y_{c}$ is not eventually periodic. Since $y_{c} \in$ $\operatorname{supp}(\omega(c))$, along with Lemma 2.4 and (2.12) this implies that

$$
\lim _{n \rightarrow \infty} \frac{1}{n} \log \left|\left(f^{n}\right)^{\prime}\left(y_{c}\right)\right|=\chi_{\mu_{c}}>0 .
$$

It is not difficult to demonstrate (see [14] for instance) that there exists $R>0$ such that for every $n \geq 1$ the holomorphic inverse branch $f_{y_{c}}^{-n}$ : $B\left(f^{n}\left(y_{c}\right), 4 R\right) \rightarrow \overline{\mathbb{C}}$ of $f^{n}$ sending $f^{n}\left(y_{c}\right)$ to $y_{c}$ is well defined. Since by Corollary 6.2 of [17] and by Koebe's distortion theorem,

$$
\lim _{n \rightarrow \infty} \frac{1}{n} \operatorname{diam}\left(f_{y_{c}}^{-n}\left(B\left(f^{n}\left(y_{c}\right), 2 R\right)\right)\right)=0,
$$

and since the point $y_{c}$ is recurrent, there exists a sequence $\left\{n_{k}\right\}_{k \geq 1}$ of positive integers increasing to infinity such that

$$
f_{y_{c}}^{-n_{k}}\left(\bar{B}\left(f^{n_{k}}\left(y_{c}\right), R\right)\right) \subset \bar{B}\left(f^{n_{k}}\left(y_{c}\right), R\right) .
$$

Therefore, by Brouwer's fixed point theorem for every $k \geq 1$ there exists a point $x_{k} \in \bar{B}\left(f^{n_{k}}\left(y_{c}\right), R\right)$ such that $f_{y_{c}}^{-n_{k}}\left(x_{k}\right)=x_{k}$. This implies that $f^{n_{k}}\left(x_{k}\right)=x_{k}$ and by Koebe's distortion theorem,

$$
\begin{aligned}
\left|\left(f^{n_{k}}\right)^{\prime}\left(x_{k}\right)\right|^{-1} & =\left|\left(f_{y_{c}}^{-n_{k}}\right)^{\prime}\left(x_{k}\right)\right| \geq K^{-1}\left|\left(f_{y_{c}}^{-n_{k}}\right)^{\prime}\left(f_{y_{c}}^{n_{k}}\left(y_{c}\right)\right)\right| \\
& =K^{-1}\left|\left(f^{n_{k}}\right)^{\prime}\left(y_{c}\right)\right|^{-1},
\end{aligned}
$$

where $K \geq 1$ is the Koebe constant corresponding to the Koebe factor $1 / 2$. Since $\lim _{k \rightarrow \infty} x_{k}=y_{c}$ and all the points $x_{k}$ are different from $y_{c}$, infinitely many of them are mutually distinct and (since these are periodic) their forward trajectory is disjoint from $\left\{y_{a}: a \in \operatorname{Crit}(f)\right\}$. Hence, for every 
$k \geq 1$ there exists $j_{k} \geq 1$ such that $x_{k} \in K\left(V_{j_{k}}\right)$. Therefore

$$
\widehat{\mathrm{P}}(t) \geq \mathrm{P}_{j_{k}}(t) \geq \mathrm{h}_{\nu_{k}}(f)-t \int \log \left|f^{\prime}\right| d \nu_{k}=\frac{-t}{n_{k}} \log \left|\left(f^{n_{k}}\right)^{\prime}\left(x_{k}\right)\right|,
$$

where $\nu_{k}$ is the ergodic $f$-invariant probability measure equidistributed on the forward orbit of $x_{k}$. Thus, it follows from (2.13) that

$$
e^{n_{k} \widehat{\mathrm{P}}(t)}\left|\left(f^{n_{k}}\right)^{\prime}\left(y_{c}\right)\right|^{t} \geq e^{n_{k} \widehat{\mathrm{P}}(t)} K^{-t}\left|\left(f^{n_{k}}\right)^{\prime}\left(x_{k}\right)\right|^{t} \geq K^{-t} .
$$

So, applying (2.11) we obtain

$$
1 \geq m\left(\left\{f^{n_{k}}\left(y_{c}\right): k \geq 1\right\}\right) \geq \sum_{k=1}^{\infty} K^{-t} m\left(y_{c}\right),
$$

which implies that $m\left(y_{c}\right)=0$. Replacing in the above considerations $y_{c}$ by $f\left(y_{c}\right)$, we see that also $m\left(f\left(y_{c}\right)\right)=0$, and in particular

$$
m\left(f\left(y_{c}\right)\right)=\int_{y_{c}} e^{\widehat{\mathrm{P}}(t)}\left|f^{\prime}\right|^{t} d m .
$$

Consequently, (2.10) holds for every Borel set $A \subset J(f)$ such $\left.f\right|_{A}$ is 1-to-1. Thus $\mathrm{P}_{\mathrm{c}}(t) \leq \widehat{\mathrm{P}}(t) \leq \mathrm{P}_{\mathrm{DU}}(t)$, which completes the proof of part (2.6) and the whole item (a) of Theorem 2.6.

In order to prove (b) notice that it follows immediately from Theorem 2.1 of [1] and Koebe's distortion theorem that if $\Omega=\emptyset$, then there are constants $C>0$ and $\beta>1$ such that for every $z \in J(f) \backslash \overline{\bigcup_{n \geq 0} f^{n}(\operatorname{Crit}(f))}$, every $n \geq 1$ and every $x \in f^{-n}(z)$, we have $\left|\left(f^{n}\right)^{\prime}(x)\right| \geq C \beta^{n}$. Write $t$ in the form $\delta+\eta, \eta>0$. Then for every $n \geq 1$,

$$
\begin{aligned}
\sum_{x \in f^{-n}(z)}\left|\left(f^{n}\right)^{\prime}(x)\right|^{-(\delta+\eta)} & =\sum_{x \in f^{-n}(z)}\left|\left(f^{n}\right)^{\prime}(x)\right|^{-\eta}\left|\left(f^{n}\right)^{\prime}(x)\right|^{-\delta} \\
& \leq C^{-\eta} \beta^{-\eta n} \sum_{x \in f^{-n}(z)}\left|\left(f^{n}\right)^{\prime}(x)\right|^{-\delta} .
\end{aligned}
$$

Hence

$$
\begin{aligned}
\mathrm{P}_{\mathrm{p}}(t) & \leq \limsup _{n \rightarrow \infty} \frac{1}{n} \log \left(C^{-\eta}\right)-\eta \log \beta+\lim _{n \rightarrow \infty} \frac{1}{n} \sum_{x \in f^{-n}(z)}\left|\left(f^{n}\right)^{\prime}(x)\right|^{-\delta} \\
& =-\eta \log \beta+\mathrm{P}_{\mathrm{p}}(\delta) \leq-\eta \log \beta<0 .
\end{aligned}
$$

Let us finally prove (c). If $\omega \in \Omega$, let $\nu$ be the purely atomic probability measure equidistributed on the forward orbit of $\omega$. The measure $\nu$ is $f$-invariant and ergodic. It follows from item (a) of our theorem that for each $t \geq 0$, $\mathrm{P}(t)=\mathrm{P}_{\mathrm{var}}(t) \geq \mathrm{h}_{\nu}-t \log \left|f^{\prime}\right| d \mu=0-0=0$. The proof of Theorem 2.6 is complete.

Remark 2.7. As Feliks Przytycki has pointed out to me, the equality $\mathrm{P}_{\mathrm{p}}(t)=\mathrm{P}_{\mathrm{var}}(t)$ follows easily for all rational functions and all $t \geq 0$ from the results proven in [13]. 
3. Conformal and invariant measures. Throughout the entire section, as in the previous one, $f: \overline{\mathbb{C}} \rightarrow \overline{\mathbb{C}}$ is assumed to be a critically nonrecurrent rational function. Given $t \geq 0$, a Borel probability measure $m_{t}$ supported on the Julia set $J(f)$ is called a t-conformal Gibbs state if $f$ is non-singular with respect to $m_{t}$ and moreover

$$
\frac{d\left(m_{t} \circ f\right)}{d m_{t}}=e^{\mathrm{P}(t)}\left|f^{\prime}\right|^{t} .
$$

It follows from Theorem 2.6 that a $t$-conformal Gibbs state exists for all $t \geq 0$. Since no critical point in the Julia set is periodic, for every $c \in$ $\operatorname{Crit}(J(f)):=\operatorname{Crit}(f) \cap J(f)$ there exists $p(c) \geq 1$ such that $f^{p(c)-1} \in$ $\operatorname{Crit}(J(f))$ and

$$
\left\{f^{j}(c): j \geq p(c)\right\} \cap \operatorname{Crit}(f)=\emptyset .
$$

Let

$$
\chi(c)=\liminf _{k \rightarrow \infty} \frac{1}{k} \log \inf _{n \geq 1}\left\{\left|\left(f^{k}\right)^{\prime}\left(f^{n}(c)\right)\right|\right\}, \quad \chi:=\min \left\{\frac{\chi(c)}{q(c)}: c \in \operatorname{Crit}(f)\right\},
$$

where $q(c)$ is the order of the critical point $c$ for the function $f^{p(c)}$. We have the following.

Proposition 3.1. If $f: \overline{\mathbb{C}} \rightarrow \overline{\mathbb{C}}$ is a critically non-recurrent rational function, then

$$
m_{t}\left(\overline{\bigcup_{n \geq 1} f^{n}(\operatorname{Crit}(J(f)))}\right)=0
$$

for each $t$-conformal Gibbs state $m_{t}$. If in addition $t \in[0, \mathrm{HD}(J(f))]$ (the case $t=\operatorname{HD}(J(f))$ is established in $[17])$, then $m_{t}(\Omega)=0$.

Proof. Since the set $\overline{\bigcup_{n>1} f^{n}(\operatorname{Crit}(J(f)))}$ is nowhere dense in $J(f)$, the desired equality can be proved in the same way as Corollary 7.2 and Lemma 7.1 in [17]. For an elaborated argument in a more complex situation see $[16]$.

The same reasoning as in Theorem 4.2 of [18] gives the following.

TheOREM 3.2. If $m_{t}$ is a t-conformal Gibbs state and if $m_{t}(\Omega)=0$, then up to a multiplicative constant there exists a unique $f$-invariant $\sigma$ finite measure $\mu_{t}$ absolutely continuous with respect to $m_{t}$. Moreover $\mu_{t}$ is equivalent to $m_{t}$ and it is conservative and ergodic.

The measure $\mu_{t}$ will be frequently called an invariant $t$-conformal Gibbs state. The critical question for our purposes is when the measure $\mu_{t}$ is finite. In order to give at least a partial answer to this question we need to know how the measure $\mu_{t}$ is constructed. This is done in the paper [8]. So, let us describe the relevant theorem and construction from that paper. Suppose that $X$ is a $\sigma$-compact metric space, $m$ is a Borel probability measure on $X$, 
positive on open sets, and that a measurable map $T: X \rightarrow X$ is given with respect to which the measure $m$ is quasi-invariant, i.e. $m \circ T^{-1} \prec m$. Moreover we assume the existence of a countable partition $\alpha=\left\{A_{n}: n \geq 0\right\}$ of $X$ into subsets which are all $\sigma$-compact and of positive $m$-measure. We also assume that $m\left(X \backslash \bigcup_{n \geq 0} A_{n}\right)=0$, and if additionally for all $m, n \geq 1$ there exists $k \geq 0$ such that

$$
m\left(T^{-k}\left(A_{m}\right) \cap A_{n}\right)>0,
$$

then the partition $\alpha$ is called irreducible. Martens's result comprising Proposition 2.6 and Theorem 2.9 of [8] reads as follows.

TheOREm 3.3. Suppose that $\alpha=\left\{A_{n}: n \geq 0\right\}$ is an irreducible partition for $T: X \rightarrow X$. Suppose that $T$ is conservative and ergodic with respect to the measure $m$. If for every $n \geq 1$ there exists $K_{n} \geq 1$ such that for all $k \geq 0$ and all Borel subsets $A$ of $A_{n}$,

$$
K_{n}^{-1} \frac{m(A)}{m\left(A_{n}\right)} \leq \frac{m\left(T^{-k}(A)\right)}{m\left(T^{-k}\left(A_{n}\right)\right)} \leq K_{n} \frac{m(A)}{m\left(A_{n}\right)}
$$

then $T$ has a $\sigma$-finite $T$-invariant measure $\mu$ absolutely continuous with respect to $m$. Additionally $\mu$ is equivalent to $m$, conservative and ergodic, and unique up to a multiplicative constant.

Since in what follows we make use of the method in which the invariant measure claimed in Theorem 3.3 is produced, we shall also describe this procedure briefly. Following Martens one considers the following sequences of measures:

$$
S_{k}(m)=\sum_{i=0}^{k-1} m \circ T^{-i} \quad \text { and } \quad Q_{k}(m)=\frac{S_{k}(m)}{S_{k}(m)\left(A_{0}\right)} .
$$

It is proven in [8] that each weak limit $\mu$ of the sequence $Q_{n}(m)$ has the properties required in Theorem 3.3, where a sequence $\left\{\nu_{k}: k \geq 1\right\}$ of measures on $X$ is said to converge weakly if for all $n \geq 1$ the measures $\nu_{k}$ converge weakly on all compact subsets of $A_{n}$. In fact it turns out that the sequence $Q_{n}(m)$ converges and

$$
\mu(F)=\lim _{n \rightarrow \infty} Q_{n}(m)(F)
$$

for every Borel set $F \subset X$. Let us now describe the construction of a partition $\alpha$ in the context of critically non-recurrent rational functions. Set $Y=J(f) \backslash(\mathrm{PC}(f) \cup \Omega)$, where $\mathrm{PC}(f)=\overline{\bigcup_{n \geq 1} f^{n}(\operatorname{Crit}(f))}$. For every $y \in Y$ consider a ball $B(y, r(y))$ such that $r(y)>0, m(\partial B(y, r(y)))=0$, and $r(y)<\frac{1}{2} \operatorname{dist}(y, \mathrm{PC}(f) \cup \Omega)$. The balls $B(y, r(y)), y \in Y$, cover $Y$ and since $Y$ is a metric separable space, one can choose a countable subcover, say $\left\{\widetilde{A}_{n}: n \geq 0\right\}$. We may additionally require that the family $\left\{\widetilde{A}_{n}: n \geq 0\right\}$ is locally finite, that is, each $x \in Y$ has an open neighborhood intersecting only 
finitely many balls $\widetilde{A}_{n}, n \geq 0$. We now define the family $\alpha=\left\{A_{n}: n \geq 0\right\}$ inductively setting

$$
A_{0}=\widetilde{A}_{0} \quad \text { and } \quad A_{n+1}=\widetilde{A}_{n}+1 \backslash \bigcup_{k=1}^{n} \overline{\widetilde{A}}_{n}
$$

(and throwing away empty sets). Obviously $\alpha$ is a disjoint family and

$$
\bigcup_{n \geq 1} A_{n} \supset J(f) \backslash(\mathrm{PC}(f) \cup \Omega) \backslash \bigcup_{n \geq 0} \partial \widetilde{A}_{n},
$$

whence in view of Corollary 7.2 in [17], $m\left(\bigcup_{n \geq 0} A_{n}\right)=1$.

In order to provide some sufficient conditions for the measure $\mu_{t}$ to be finite, we need an assumption stronger than critical non-recurrence. Namely, from now on throughout this section we assume that $f: \overline{\mathbb{C}} \rightarrow \overline{\mathbb{C}}$ is parabolically semi-hyperbolic, that is, $c \notin \omega(\operatorname{Crit}(f))$ for all $c \in \operatorname{Crit}(J(f))$ and $\omega(\operatorname{Crit}(J(f))) \cap \Omega(f)=\emptyset$.

TheOREM 3.4. Suppose that $f: \overline{\mathbb{C}} \rightarrow \overline{\mathbb{C}}$ is a parabolically semi-hyperbolic map and that $\mathrm{P}(t)>-\chi t$. If $m_{t}$ is the t-conformal Gibbs state and $m_{t}(\Omega)=0$, then the set of points of infinite condensation of the invariant $t$-conformal Gibbs state $\mu_{t}$ is contained in $\Omega(f)$.

Proof. By the standard normal family argument there exist $u \geq 1$ and $\lambda>1$ such that

$$
\left|\left(f^{u}\right)^{\prime}(z)\right|>\lambda
$$

for all $z \in \omega(\operatorname{Crit}(J(f)))$. Thus

$$
\left|\left(f^{u}\right)^{\prime}\left(f^{n}(c)\right)\right| \geq \lambda
$$

for every $c \in \operatorname{Crit}(J(f))$ and all $n$ large enough. Since the conformal measure $m_{t}$ is positive on non-empty open sets, $\inf \left\{m_{t}(B(x, r)): x \in J(f)\right\}>0$ for every $r>0$; even more, there exists $\alpha(r) \in(0, r)$ such that

$$
M(r)=\inf \left\{m_{t}(B(x, r) \backslash B(x, \alpha(r))): x \in J(f)\right\}>0 .
$$

It follows from (3.1) and (3.2) that there exists $\delta>0$ such that for every $c \in \operatorname{Crit}(J(f)), k \geq 1$ and every $n \geq p(c)$ the holomorphic inverse branch $f_{f^{n}(c)}^{-k}: B\left(f^{n+k}(c), 4 \delta\right) \rightarrow \mathbb{C}$ sending $f^{n+k}(c)$ to $f^{n}(c)$ is well defined. It also follows from (3.2) that for all $u$ large enough, all $c \in \operatorname{Crit}(J(f))$, all $k \geq 0$ and all $0 \leq i \leq u-1$,

$$
f_{f^{p(c)+i+k u}}^{-u}\left(B\left(f^{p(c)+i+(k+1) u}(c), 2 \delta\right)\right) \subset B\left(f^{p(c)+i+k u}(c), \alpha(\delta)\right) .
$$

For every $c \in \operatorname{Crit}(J(f))$, all $0 \leq j \leq u-1$ and all $i \geq 0$ define now

$$
\begin{aligned}
R_{i, j}(c) & =f_{f^{p+i}(c)}^{-j u}\left(B\left(f^{p+i+j u}(c), 2 \delta\right)\right) \backslash f_{f^{p+i}(c)}^{-(j+1) u}\left(B\left(f^{p+i+(j+1) u}(c), 2 \delta\right)\right) \\
& =f_{f^{p+i}(c)}^{-j u}\left(B\left(f^{p+i+j u}(c), 2 \delta\right) \backslash f_{f^{p+i+j u}(c)}^{-u}\left(B\left(f^{(p+i+(j+1) u}(c), 2 \delta\right)\right)\right),
\end{aligned}
$$


where $p=p(c)$. Applying Koebe's distortion theorem and using (3.3) along with (3.4) we conclude that

$$
\begin{aligned}
m_{t}\left(R_{i, j}(c)\right) \asymp e^{-\mathrm{P}(t) j u}\left|\left(f^{j u}\right)^{\prime}\left(f^{p+i}(c)\right)\right|^{-t} \\
\quad \times m_{t}\left(B\left(f^{p+i j u}(c), 2 \delta\right) \backslash f_{f^{p+i+j u}(c)}^{-u}\left(B\left(f^{(p+i+(j+1) u}(c), 2 \delta\right)\right)\right) \\
\asymp e^{-\mathrm{P}(t) j u}\left|\left(f^{j u}\right)^{\prime}\left(f^{p+i}(c)\right)\right|^{-t} .
\end{aligned}
$$

Fix now a point $\xi \in \overline{\bigcup_{n \geq 0} f^{n}(\operatorname{Crit}(J(f)))}$. Obviously, because of parabolical semi-hyperbolicity of $f$, the latter set is disjoint from $\Omega$. Let $x=f^{s}(\xi)$, where $s \geq 1$ is so large that

$$
\left\{f^{n}(x): n \geq 0\right\} \cap \operatorname{Crit}(f)=\emptyset .
$$

Since $\operatorname{Crit}(J(f)) \cap \omega(\operatorname{Crit}(J(f)))=\emptyset$, it follows from Lemma 2.13 in [17] that there exists $0<\gamma<\delta / 2$ such that if $n \geq 1$ and $y \in f^{-n}(x)$, then $f^{k}\left(C_{n}(y, B(x, 4 \gamma))\right) \cap \operatorname{Crit}(f)$ consists of at most one point for every $0 \leq k \leq n-1$. Without loss of generality we may assume that the set $A_{0}$ involved in the definition of the invariant measure $\mu_{t}$ is contained in $B(x, \gamma)$. Suppose first that $C_{n}(y, B(x, 2 \gamma)) \cap \operatorname{Crit}\left(f^{n}\right)=\emptyset$. It then follows from Koebe's distortion theorem that

$$
\frac{m_{t}\left(C_{n}(y, B(x, \gamma))\right)}{m_{t}\left(C_{n}(y, B(x, \gamma)) \cap f^{-n}\left(A_{0}\right)\right)} \preceq \frac{m_{t}(B(x, \gamma)}{m_{t}\left(A_{0}\right)} \asymp 1 .
$$

Suppose in turn that $C_{n}(y, B(x, 2 \gamma)) \cap \operatorname{Crit}\left(f^{n}\right) \neq \emptyset$ and let $0 \leq k \leq n-1$ be the least integer such that $f^{k}\left(C_{n}(y, B(x, 4 \gamma))\right) \cap \operatorname{Crit}(f)$ is not-empty. Denote by $c$ its only element. Note that by $(3.7), k+p(c) \leq n$. Put $p=p(c)$. Taking $\gamma>0$ sufficiently small, we may assume that

$$
\operatorname{Crit}(f) \cap \bigcup_{k=0}^{n} f^{k}\left(C_{n}(y, B(x, \gamma))\right) \subset\left\{f^{j}(c): 0 \leq j \leq p-1\right\} .
$$

In particular

$$
c \in f^{k}\left(C_{n}(y, B(x, 2 \gamma))\right)
$$

We have

$$
\left|\left(f^{p+i}\right)^{\prime}(z)\right| \asymp|z-c|^{q(c)-1}
$$

for all $0 \leq i \leq u-1$ and all $z \in C_{p+i}\left(c, B\left(f^{p+i}(c), 2 \delta\right)\right.$ ) (note that $q(c)$ is also the order of $c$ for the function $\left.f^{p+i}\right)$. Write $n-k-p=s u+r, s \geq 0$, $0 \leq r \leq u-1$. Since

$$
f^{k}\left(C_{n}(y, B(x, \gamma))\right) \subset C_{p+r+s u}\left(c, B\left(f^{p+r+s u}(c), \delta\right)\right),
$$

using (3.6) and (3.10), we get 


$$
\begin{aligned}
& m_{t}\left(f^{k}\left(C_{n}(y, B(x, \gamma))\right)\right) \\
& \preceq \sum_{j \geq s}\left|\left(f^{j u}\right)^{\prime}\left(f^{p+r}(c)\right)\right|^{-t} e^{-\mathrm{P}(t)(p+r+j u)}\left(\left|\left(f^{j u}\right)^{\prime}\left(f^{p+r}(c)\right)\right|^{-1}\right)^{(1 / q(c)-1) t} \\
& \asymp \sum_{j \geq s} e^{-\mathrm{P}(t)(p+r+j u)}\left|\left(f^{j u}\right)^{\prime}\left(f^{p+r}(c)\right)\right|^{-t / q(c)} .
\end{aligned}
$$

Since $A_{0} \subset B(x, \gamma) \subset B\left(f^{p+r+s u}(c), \delta\right)$, using Koebe's distortion theorem, we get

$$
\begin{aligned}
m_{t} & \left(f^{k}\left(C_{n}(y, B(x, \gamma))\right) \cap f^{-(n-k)}\left(A_{0}\right)\right) \\
& \asymp m_{t}\left(A_{0}\right)\left|\left(f^{s u}\right)^{\prime}\left(f^{p+r}(c)\right)\right|^{-t} e^{-\mathrm{P}(t)(p+r+s u)}\left(\left|\left(f^{s u}\right)^{\prime}\left(f^{p+r}(c)\right)\right|^{-1}\right)^{(1 / q(c)-1) t} \\
& \asymp e^{-\mathrm{P}(t)(p+r+s u)}\left|\left(f^{s u}\right)^{\prime}\left(f^{p+r}(c)\right)\right|^{-t / q(c)} .
\end{aligned}
$$

Therefore, using the assumption $\mathrm{P}(t)>-\chi t$, we conclude that

$$
\begin{aligned}
& \frac{m_{t}\left(f^{k}\left(C_{n}(y, B(x, \gamma))\right)\right)}{m_{t}\left(f^{k}\left(C_{n}(y, B(x, \gamma))\right) \cap f^{-(n-k)}\left(A_{0}\right)\right)} \\
& \preceq \sum_{j \geq 0} e^{-\mathrm{P}(t)(p+r+j u)}\left|\left(f^{j u}\right)^{\prime}\left(f^{p+r+s u}(c)\right)\right|^{-t / q(c)} \leq S(c)
\end{aligned}
$$

for some number $S(c)$ depending only on $c$. Using (3.9) we conclude that

$$
\left(f^{k}\left(C_{n}(y, B(x, 4 \gamma))\right) \backslash f^{k}\left(C_{n}(y, B(x, 2 \gamma))\right)\right) \cap \operatorname{Crit}\left(f^{n-k}\right)=\emptyset
$$

and therefore $\operatorname{Mod}\left(f^{k}\left(C_{n}(y, B(x, 4 \gamma))\right) \backslash f^{k}\left(C_{n}(y, B(x, 2 \gamma))\right)\right) \geq(\log 2) / q(c)$. Hence, applying Koebe's distortion theorem and (3.11), we obtain

$$
\begin{aligned}
& \frac{m_{t}\left(C_{n}(y, B(x, \gamma))\right)}{m_{t}\left(C_{n}(y, B(x, \gamma)) \cap f^{-n}\left(A_{0}\right)\right)} \\
& \quad \asymp \frac{\left|\left(f^{k}\right)^{\prime}(y)\right|^{-t} e^{-\mathrm{P}(t) k}}{\left|\left(f^{k}\right)^{\prime}(y)\right|^{-t} e^{-\mathrm{P}(t) k}} \cdot \frac{m_{t}\left(f^{k}\left(C_{n}(y, B(x, \gamma))\right)\right)}{m_{t}\left(f^{k}\left(C_{n}(y, B(x, \gamma))\right) \cap f^{-(n-k)}\left(A_{0}\right)\right)} \\
& \quad \preceq S(c) .
\end{aligned}
$$

Therefore

$$
\frac{m_{t}\left(f^{-n}(B(x, \gamma))\right.}{m_{t}\left(f^{-n}\left(A_{0}\right)\right)} \preceq \max \{S(c): c \in \operatorname{Crit}(J(f))\}
$$

and consequently $Q_{n}(B(x, \gamma)) \preceq \max \{S(c): c \in \operatorname{Crit}(J(f))\}$ for all $n \geq 1$. Thus $\mu_{t}(B(x, \gamma))<\infty$ and $x$ is a point of finite condensation of $\mu_{t}$. Since $\mu_{t}$ is an invariant measure, we conclude that $\xi$ is also a point of finite condensation of $\mu_{t}$ and we are done. If $\xi \in J(f) \backslash\left(\overline{\bigcup_{n \geq 0} f^{n}(\operatorname{Crit}(J(f)))} \cup \Omega(f)\right)$ the argument is easier.

A parabolically semi-hyperbolic map $f: \overline{\mathbb{C}} \rightarrow \overline{\mathbb{C}}$ is called semi-hyperbolic if $\Omega(f)=\emptyset$. As an immediate consequence of Theorem 3.4, we get the following. 
Corollary 3.5. Suppose $f: \overline{\mathbb{C}} \rightarrow \overline{\mathbb{C}}$ is semi-hyperbolic. If $\mathrm{P}(t)>-\chi t$, then the invariant t-conformal Gibbs state $\mu_{t}$ is finite.

Allowing parabolic points we still get the following remarkable

TheOREM 3.6. Suppose that $f: \overline{\mathbb{C}} \rightarrow \overline{\mathbb{C}}$ is parabolically semi-hyperbolic. If $t \in\left[0, \mathrm{HD}(J(f))\right.$ ) (then $m_{t}(\Omega)=0$ by Proposition $3.1, \chi>0$ follows from parabolical semi-hyperbolicity, and $\mathrm{P}(t)>0>-\chi t)$, then the $t$-conformal Gibbs state $\mu_{t}$ is finite.

Proof. Since $h=\delta(f)$ and $t \in[0, h)$, it follows from Theorem 2.2 that $\mathrm{P}(t)>0$. Hence, for every $k \geq 1$ and every $\omega \in \Omega$,

$$
\begin{aligned}
m_{t}\left(B\left(\omega, k^{-1 / p(\omega)}\right)\right) \asymp \sum_{j=k^{1 / p(\omega)}}^{\infty} e^{-\mathrm{P}(t) j} j^{-m(p(\omega)+1) t / p(\omega)} \\
\leq \sum_{j=k^{1 / p(\omega)}}^{\infty} e^{-\mathrm{P}(t) j} \asymp e^{-\mathrm{P}(t) k^{1 / p(\omega)}} .
\end{aligned}
$$

Therefore, proceeding exactly as in the proof of Proposition 6.2 from [18], instead of (6.2) we would get

$$
Q_{n}(B) \asymp \sum_{k=1}^{n-1} e^{-\mathrm{P}(t) k^{1 / p(\omega)}} \frac{S_{n-k}\left(m_{t}\right)(A)}{S_{n}\left(m_{t}\right)(A)} \leq \sum_{k=1}^{\infty} e^{-\mathrm{P}(t) k^{1 / p(\omega)}} .
$$

Since $\mathrm{P}(t)>0$, this last series converges and all the numbers $Q_{n}(B)$ are bounded above by its sum multiplied by a universal constant. This shows that $\omega$ is a point of finite condensation of $\mu_{t}$, which finishes the proof in view of Theorem 3.4 .

4. Escape rates. Throughout this section $f: \overline{\mathbb{C}} \rightarrow \overline{\mathbb{C}}$ is a critically non-recurrent rational map. Let $\lambda$ be the normalized Lebesgue measure on the sphere $\overline{\mathbb{C}}$ (with respect to the spherical metric). Following the reasoning from Lemma 5.3 of [18] we shall prove the following.

LEMMA 4.1. If $f: \overline{\mathbb{C}} \rightarrow \overline{\mathbb{C}}$ is critically non-recurrent, then for every $\theta>0$ there exists $\varepsilon>0$ such that if $D$ is an open ball centered in $J(f) \backslash B(\Omega, \theta)$ with radius $\varepsilon$ and $B$ is an open ball such that $2 B \subset D \backslash \overline{\bigcup_{n \geq 1} f^{n}(\operatorname{Crit}(f))}$, then

$$
\inf \left\{\frac{\lambda\left(B_{n}\right)}{\lambda\left(D_{n}\right)}\right\}>0
$$

where the infimum is taken over all integers $n \geq 0$, all connected components $D_{n}$ of $f^{-n}(D)$, and all connected components $B_{n}$ of $f^{-n}(B)$ contained in $D_{n}$. 
Proof. Using Koebe's distortion theorem we obtain

$$
\lambda\left(B_{n}\right) \asymp \operatorname{diam}^{2}\left(B_{n}\right)=\left(\frac{\operatorname{diam}\left(B_{n}\right)}{\operatorname{diam}\left(D_{n}\right)}\right)^{2} \operatorname{diam}^{2}\left(D_{n}\right) \geq \lambda\left(D_{n}\right)\left(\frac{\operatorname{diam}\left(B_{n}\right)}{\operatorname{diam}\left(D_{n}\right)}\right)^{2}
$$

and applying Lemma 3.3 in [18] we get

$$
\lambda\left(B_{n}\right) \succeq \lambda\left(D_{n}\right)\left(\frac{\operatorname{diam}(B)}{\operatorname{diam}(D)}\right)^{2} .
$$

THEOREM 4.2. If $f$ is critically non-recurrent, then for every $\varepsilon>0$ sufficiently small

$$
\limsup _{n \rightarrow \infty} \frac{1}{n} \log \lambda\left(f^{-n}(B(J(f), \varepsilon))\right)=\mathrm{P}(2) .
$$

Proof. Fix $\varepsilon>0$ so small as required in Lemma 4.1 and such that there exists $z \in G \backslash \overline{\bigcup_{n \geq 1} f^{n}(\operatorname{Crit}(f))}$ for which $B(z, 2 \varepsilon) \cap \overline{\bigcup_{n \geq 1} f^{n}(\operatorname{Crit}(f))}=\emptyset$ (by topological exactness of $f: J(f) \rightarrow J(f), \overline{\bigcup_{n \geq 1} f^{n}(\operatorname{Crit}(f))}$ is obviously a nowhere-dense subset of $J(f)$ in the critically non-recurrent case), where $G$ is the set coming from item 6 of the definition of topological pressures. Applying Koebe's distortion theorem we obtain

$$
\begin{aligned}
& \limsup _{n \rightarrow \infty} \frac{1}{n} \log \lambda\left(f^{-n}(B(J(f), \varepsilon))\right) \\
\geq & \limsup _{n \rightarrow \infty} \frac{1}{n} \log \lambda\left(f^{-n}(B(z, \varepsilon))\right)=\limsup _{n \rightarrow \infty} \frac{1}{n} \log \sum_{y \in f^{-n}(z)} \lambda\left(f_{y}^{-n}(B(z, \varepsilon))\right) \\
= & \limsup _{n \rightarrow \infty} \frac{1}{n} \log \sum_{y \in f^{-n}(z)}\left|\left(f^{n}\right)^{\prime}(y)\right|^{-2}=\mathrm{P}(2) .
\end{aligned}
$$

Since obviously

$$
\limsup _{n \rightarrow \infty} \frac{1}{n} \log \lambda\left(f^{-n}(B(J(f), \varepsilon))\right) \leq 0,
$$

combining this, (4.1) and Theorem 2.6(c) concludes the proof in the case when $\Omega \neq \emptyset$. If $\Omega=\emptyset$, cover $J(f)$ by balls $\left\{D_{i}\right\}_{i=1}^{k}$ centered respectively at some points $\left\{x_{i}\right\}_{i=1}^{k}$ in $G$ and with radii so small as required in Lemma 4.1. Since, as already observed, $\overline{\bigcup_{n \geq 1} f^{n}(\operatorname{Crit}(f))}$ is nowhere dense in the Julia set $J(f)$, each ball $D_{i}, i=1, \ldots, k$, contains a non-empty open ball $B_{i}$ such that $2 B_{i} \subset D_{i} \backslash \overline{\bigcup_{n \geq 1} f^{n}(\operatorname{Crit}(f))}$. Thus, applying Lemma 4.1 and Koebe's distortion theorem, we get for every $i=1, \ldots, k$,

$$
\begin{aligned}
\limsup _{n \rightarrow \infty} \frac{1}{n} \log \lambda\left(f^{-n}\left(D_{i}\right)\right) & =\limsup _{n \rightarrow \infty} \frac{1}{n} \log \lambda\left(f^{-n}\left(B_{i}\right)\right) \\
& =\limsup _{n \rightarrow \infty} \frac{1}{n} \log \sum_{y \in f^{-n}\left(x_{i}\right)}\left|\left(f^{n}\right)^{\prime}(y)\right|^{-2}=\mathrm{P}(2) .
\end{aligned}
$$


Hence, assuming $\varepsilon>0$ to be so small that $B(J(f), \varepsilon) \subset D_{1} \cup \ldots \cup D_{k}$, we get

$\limsup _{n \rightarrow \infty} \frac{1}{n} \log \lambda\left(f^{-n}(B(J(f), \varepsilon))\right) \leq \limsup _{n \rightarrow \infty} \frac{1}{n} \log \lambda\left(\bigcup_{i=1}^{k} f^{-n}\left(D_{i}\right)\right)=\mathrm{P}(2)$.

In particular, it therefore follows from Theorem 2.6(b) that if $\Omega=\emptyset$, then the rate of escape is exponential. In the case when $\Omega \neq \emptyset$, something more can be said about a modified rate of escape. Let $p(\omega) \geq 1$ be the number of petals of a parabolic point $\omega$ and let

$$
p=\max \{p(\omega): \omega \in \Omega\}, \quad \xi=\left(\prod_{c \in \operatorname{Crit}(f)} q(c)\right)^{-1},
$$

where $q(c) \geq 2$ is the order of the critical point $c$ of $f$. Let $\theta$ have the same meaning as in [18]. We will need the following.

LEMMA 4.3. If $f$ is critically non-recurrent, then there exists a constant $B \geq 1$ such that if $\varepsilon>0$ is small enough, $n \geq 1$ is an integer, $z \in J(f)$, and $f^{n}(z) \notin B(\Omega, \theta)$, then

$$
\operatorname{diam}\left(C_{n}\left(z, B\left(f^{n}(z), \varepsilon\right)\right)\right) \leq B n^{-\xi(p+1) / p} .
$$

Proof. Since

$\lim _{n \rightarrow \infty} \sup \left\{\operatorname{diam}\left(C_{n}\left(w, B\left(f^{n}(w), \varepsilon\right)\right)\right): w \in J(f), f^{n}(w) \notin B(\Omega, \theta)\right\}=0$,

there exists $q \geq 1$ such that for every $n \geq q, w \in J(f), f^{n}(w) \notin B(\Omega, \theta)$, we have

$$
\operatorname{diam}\left(C_{n}\left(w, B\left(f^{n}(w), \varepsilon\right)\right)\right)<\varepsilon / 4 \text {. }
$$

Suppose now that $n \geq q+1$. We shall inductively define the sequence

$$
x_{0}=f^{k_{0}}(z), x_{1}=f^{k_{1}}(z), \ldots, x_{l}=f^{k_{l}}(z)
$$

$(l \leq n)$ of points from the set $\left\{z, f(z), \ldots, f^{n}(z)\right\}$ as follows. We declare $k_{0}=n$ and $x_{0}=f^{n}(z)$. If all other points from $\left\{z, f(z), \ldots, f^{n}(z)\right\}$ are contained in $B(\Omega, \theta)$, we put $x_{1}=z$ and stop the inductive procedure. Assume now that $x_{j}=f^{k_{j}}(z) \in\left\{z, f(z), \ldots, f^{n}(z)\right\} \backslash B(\Omega, \theta)$ has been defined for all $j \in\{0,1, \ldots, n\}$. If $k_{j}<q$, we stop the inductive procedure. If $\left\{z, f(z), \ldots, f^{k_{j}-q}(z)\right\} \subset B(\Omega, \theta)$, we stop the inductive procedure by setting $x_{j+1}=z$. Otherwise we define $x_{j+1}=f^{k}(z)$, where $0 \leq k \leq k_{j}-q$ is the largest integer such that $f^{k}(z) \notin B(\Omega, \theta)$. For every $0 \leq j \leq l-1$ define first the sets

$$
C_{j}=C_{k_{j}-k_{j+1}}\left(x_{j+1}, B\left(x_{j}, \varepsilon\right)\right) \quad \text { and } \quad B_{j}=C_{k_{j}}\left(z, B\left(x_{j}, \varepsilon\right)\right) .
$$

Since $k_{j}-k_{j+1} \geq q$, we have $C_{j} \subset B\left(x_{j+1}, \varepsilon / 2\right)$ and define the set

$$
A_{j}=C_{k_{j+1}}\left(z, B\left(x_{j+1}, \varepsilon\right)\right) \backslash C_{k_{j+1}}\left(z, C_{j}\right)=B_{j+1} \backslash B_{j} .
$$

Since $C_{j} \subset B\left(x_{j+1}, \varepsilon / 2\right)$, we obtain $B_{j} \subset B_{j+1}$ if $j \leq l-1$ and

$$
A_{j} \supset C_{k_{j+1}}\left(B\left(x_{j+1}, \varepsilon\right) \backslash C_{j}\right)
$$


where $C_{k_{j+1}}\left(B\left(x_{j+1}, \varepsilon\right) \backslash C_{j}\right)$ is the connected component of the set $f^{-k_{j+1}}\left(B\left(x_{j+1}, \varepsilon\right) \backslash C_{j}\right)$ that encloses $B_{j}$ in $B_{j+1}$. Improving slightly Lemma 3.1 of [18] we get

$$
\operatorname{Mod}\left(A_{j}\right) \geq \xi \log 2 .
$$

It follows from the local behavior of $f$ around parabolic points and the definition of the sequence $\left\{x_{j}\right\}_{j=0}^{l}$ that there exists a constant $L \leq 1$ such that for all $0 \leq j \leq l-1$,

$$
\operatorname{diam}\left(C_{j}\right) \leq\left(L\left(k_{j}-k_{j+1}\right)\right)^{-(p+1) / p} .
$$

Fix now an integer $u \geq 1$ so large that for every $t \geq 2$,

$$
\frac{t e^{M t}}{u^{t-1}} \leq 1, \quad \text { where } \quad M=-\left(\log L+\frac{p}{p+1} \log \varepsilon\right) .
$$

We define

$$
R=\left\{j \in\left\{0,1, \ldots, l-1: k_{j}-k_{j+1} \geq u\right\} .\right.
$$

It then follows from (4.5) that for all $j \in R$,

$$
\begin{aligned}
\operatorname{Mod}\left(B\left(x_{j+1}, \varepsilon\right) \backslash C_{j}\right) & \geq \log \varepsilon-\log \operatorname{diam}\left(C_{j}\right) \\
& \geq \log \varepsilon+\frac{p+1}{p} \log L+\frac{p+1}{p} \log \left(k_{j}-k_{j+1}\right) \\
& =\frac{p+1}{p}\left(\log \left(k_{j}-k_{j+1}\right)-M\right) .
\end{aligned}
$$

Proceeding in the same way as in the proof of Lemma 3.1 in [18] and using (4.3), we deduce that if $j \in R$, then

$$
\operatorname{Mod}\left(A_{j}\right) \geq \xi \frac{p+1}{p}\left(\log \left(k_{j}-k_{j+1}\right)-M\right)
$$

Combining this with (4.4) and using the Grötzsch inequality, we conclude that

$$
\begin{aligned}
& \operatorname{Mod}\left(B(z, \varepsilon) \backslash C_{n}\left(z, B\left(f^{n}(z), \varepsilon\right)\right)\right) \\
& \quad \geq \xi \log 2(l-1-\# R)+\xi \frac{p+1}{p}\left(\sum_{j \in R} \log \left(k_{j}-k_{j+1}\right)-M \# R\right) .
\end{aligned}
$$

If $\# R \geq 2$, then using (4.6) we obtain

$$
\begin{aligned}
& \frac{\exp \left(-\xi \frac{p+1}{p}\left(\sum_{j \in R} \log \left(k_{j}-k_{j+1}\right)-M \# R\right)\right)}{\left(\sum_{j \in R}\left(k_{j}-k_{j+1}\right)\right)^{-\xi(p+1) / p}} \\
= & \left(e^{M \# R} \frac{\sum_{j \in R}\left(k_{j}-k_{j+1}\right)}{\prod_{j \in R}\left(k_{j}-k_{j+1}\right)}\right)^{\xi(p+1) / p} \\
= & \left(e^{M \# R} \sum_{j \in R} \frac{1}{\prod_{i \in R \backslash\{j\}}\left(k_{i}-k_{i+1}\right)}\right)^{\xi(p+1) / p} \leq\left(\frac{\# R e^{M \# R}}{u^{\# R-1}}\right)^{\xi(p+1) / p} \leq 1 .
\end{aligned}
$$


Since, by our inductive construction,

$$
l-1-\# R \geq \frac{1}{q}\left(n-q-\sum_{j \in R}\left(k_{j}-k_{j+1}\right)\right) \geq \frac{1}{2 q}\left(n-\sum_{j \in R}\left(k_{j}-k_{j+1}\right)\right)
$$

for all $n$ large enough, we therefore obtain

$$
\begin{aligned}
& \exp \left(-\operatorname{Mod}\left(B(z, \varepsilon) \backslash C_{n}\left(z, B\left(f^{n}(z), \varepsilon\right)\right)\right)\right) \\
& \leq \exp \left(\frac{-\xi \log 2}{2 q}\left(n-\sum_{j \in R}\left(k_{j}-k_{j+1}\right)\right)\right)\left(\sum_{j \in R}\left(k_{j}-k_{j+1}\right)\right)^{-\xi(p+1) / p} \\
& \leq B_{1} n^{-\xi(p+1) / p}
\end{aligned}
$$

for a sufficiently large constant $B_{1}$. Since in the case $\# R \leq 1,(4.10)$ follows immediately from (4.8), the formula (4.10) is always true. In view of Theorem 2.4 of [11], the hyperbolic diameter of $C_{n}\left(z, B\left(f^{n}(z), \varepsilon\right)\right)$ in $B(z, \varepsilon)$ is bounded above by

$$
B_{2} \exp \left(-\operatorname{Mod}\left(B(z, \varepsilon) \backslash C_{n}\left(z, B\left(f^{n}(z), \varepsilon\right)\right)\right)\right) \leq B_{2} B_{1} n^{-\xi(p+1) / p}
$$

for some universal constant $B_{2}$, and the inequality sign was written due to (4.10). Since $z \in C_{n}\left(z, B\left(f^{n}(z), \varepsilon\right)\right)$ and $\lim _{n \rightarrow \infty} B_{2} B_{1} n^{-\xi(p+1) / p}=0$, the euclidean and hyperbolic diameters of $C_{n}\left(z, B\left(f^{n}(z), \varepsilon\right)\right)$ become comparable, and consequently there exists a constant $B \geq 1$ such that

$$
\operatorname{diam}\left(C_{n}\left(z, B\left(f^{n}(z), \varepsilon\right)\right)\right) \leq B n^{-\xi(p+1) / p}
$$

for all $n \geq 1$.

REMARK 4.4. In the proof of Lemma 4.3 one shows in fact that $\operatorname{Mod}\left(A_{j}\right)$ $\geq \xi_{n}$, where $\xi_{n}$ is the degree of $f^{n}$ restricted to $C_{n}\left(z, B\left(f^{n}(z), \varepsilon\right)\right)$. In particular if $C_{n}\left(z, B\left(f^{n}(z), \varepsilon\right)\right)$ contains no critical points of $f^{n}$, then $\xi_{n}=1$ and

$$
\operatorname{diam}\left(C_{n}\left(z, B\left(f^{n}(z), \varepsilon\right)\right)\right) \leq B n^{(p+1) / p} .
$$

So, in the parabolic case (no critical points in the Julia sets), we get the result proven in [6].

Recall that in view of Theorem 2.6, $h=\mathrm{HD}(J(f))$ and $\mathrm{P}(h)=0$. Developing now the approach from [6] we shall prove the following.

THEOREM 4.5. If $f$ is a critically non-recurrent rational function, then for every $0<\delta<\operatorname{diam}(J(f))$ there exist constants $\varepsilon>0$ and $C \geq 1$ such that

$$
C^{-1} n^{-2(p+1) / p} \leq \lambda\left(f^{-n}(B(J(f) \backslash B(\Omega, \delta), \varepsilon))\right) \leq C n^{-\xi(h-2)(p+1) / p} .
$$

Proof. Fix $\omega \in \Omega$ such that $p(\omega)=p$. Let $q \geq 1$ be the period of $\omega$. Fix also $y \in B(J(f) \backslash B(\Omega, \delta), \varepsilon) \backslash \overline{\bigcup_{n \geq 1} f^{n}(\operatorname{Crit}(f))}$ and then $x \in f^{-l}(y)$ for some $l \geq 1$, so close to $\omega$ that the inverse branches $f_{\omega}^{-q k}$ of $f^{q k}$ are well defined on 
some neighborhood $B(x, r)$ of $x$ and $\lim _{k \rightarrow \infty} f_{\omega}^{-q k}(B(x, r))=\omega$. Take then $0<\eta<\varepsilon$ so small that $B(y, \eta) \subset B(J(f) \backslash B(\Omega, \delta), \varepsilon)$ and $f_{x}^{-l}(B(y, \eta)) \subset$ $B(x, r)$. It follows from the local behavior of $f$ around parabolic points that for every $k \geq 1$,

$$
\begin{aligned}
\lambda\left(f^{-(l+q k)}(B(J(f) \backslash B(\Omega, \delta), \varepsilon))\right) & \geq \lambda\left(f_{\omega}^{-q k}(B(x, r))\right) \\
& \asymp\left|\left(f_{\omega}^{-q k}\right)^{\prime}(x)\right|^{2} \asymp(q k)^{-2(p+1) / p}
\end{aligned}
$$

and the first inequality of Theorem 4.5 easily follows. In order to prove the opposite inequality fix $\kappa>0$ ascribed to $\delta>0$ so small as required in Lemma 4.1 and cover $J(f) \backslash B(\Omega, \delta)$ with finitely many open balls $D_{1}, \ldots, D_{k}$ with radii $\kappa$ centered at points of the set $J(f) \backslash B(\Omega, \delta)$. Since $J(f) \backslash B(\Omega, \delta)$ is a compact set, there exists $\varepsilon>0$ such that

$$
B(J(f) \backslash B(\Omega, \delta), \varepsilon) \subset D_{1} \cup \ldots \cup D_{k} .
$$

For every $i \in\{1, \ldots, k\}$ fix then an open ball $B_{i}$ such that $2 B_{i} \subset D_{i} \backslash$ $\overline{\bigcup_{n \geq 1} f^{n}(\operatorname{Crit}(f))}$ and denote its center by $z_{i}$. Now, applying Koebe's distortion theorem and Lemma 4.3 (with $\delta=\theta$ ), for every $i \in\{1, \ldots, k\}$ and for every $n \geq 1$ we obtain

$$
\begin{aligned}
& \lambda\left(f^{-n}\left(B_{i}\right)\right) \\
& =\lambda\left(\bigcup_{x \in f^{-n}\left(z_{i}\right)} f_{x}^{-n}\left(B_{i}\right)\right) \leq \sum_{x \in f^{-n}\left(z_{i}\right)} \lambda\left(f_{x}^{-n}\left(B_{i}\right)\right) \\
& \asymp \sum_{x \in f^{-n}\left(z_{i}\right)}\left|\left(f^{n}\right)^{\prime}(x)\right|^{-2} \lambda\left(B_{i}\right) \\
& \asymp \lambda\left(B_{i}\right) \sum_{x \in f^{-n}\left(z_{i}\right)}\left|\left(f^{n}\right)^{\prime}(x)\right|^{h-2}\left|\left(f^{n}\right)^{\prime}(x)\right|^{-h} \\
& \asymp \lambda\left(B_{i}\right) \sum_{x \in f^{-n}\left(z_{i}\right)} \operatorname{diam}\left(C_{n}\left(x, B\left(z_{i}, r\right)\right)\right)^{2-h}\left|\left(f^{n}\right)^{\prime}(x)\right|^{-h} \\
& \preceq \lambda\left(B_{i}\right) \sum_{x \in f^{-n}\left(z_{i}\right)} n^{\xi(h-2)(p+1) / p}\left|\left(f^{n}\right)^{\prime}(x)\right|^{-h} \\
& =\lambda\left(B_{i}\right) n^{\xi(h-2)(p+1) / p} \sum_{x \in f^{-n}\left(z_{i}\right)}\left|\left(f^{n}\right)^{\prime}(x)\right|^{-h} \\
& \asymp \lambda\left(B_{i}\right) n^{\xi(h-2)(p+1) / p} \sum_{x \in f^{-n}\left(z_{i}\right)} m_{h}\left(f_{x}^{-n}\left(B\left(z_{i}, r\right)\right)\right) \\
& \asymp \lambda\left(B_{i}\right) n^{\xi(h-2)(p+1) / p} m_{h}\left(f^{-n}\left(B\left(z_{i}, r\right)\right)\right) \preceq \lambda\left(B_{i}\right) n^{\xi(h-2)(p+1) / p} .
\end{aligned}
$$

Applying now Lemma 4.1 and (4.11), for every $n \geq 1$ we obtain 


$$
\begin{aligned}
& \lambda\left(f^{-n}(\right.B(J(f) \backslash B(\Omega, \delta), \varepsilon))) \\
& \quad \leq \sum_{i=1}^{k} \lambda\left(f^{-n}\left(D_{i}\right)\right) \preceq \sum_{i=1}^{k} \lambda\left(f^{-n}\left(B_{i}\right)\right) \preceq \sum_{i=1}^{k} \lambda\left(B_{i}\right) n^{\xi(h-2)(p+1) / p} \\
& \quad \leq k \max \left\{\lambda\left(B_{i}\right): i \in\{1, \ldots, k\}\right\} n^{\xi(h-2)(p+1) / p} . \text {. }
\end{aligned}
$$

Since in the parabolic case (no critical points in the Julia sets) $\xi=1$, as an immediate consequence of Theorem 4.5 we get the corresponding result proven in [6].

5. Real-analyticity of topological pressure. This section differs from the previous sections in two points. We consider the generalized polynomial-like mappings (defined below) instead of rational functions, and we consider Hölder continuous potentials instead of the functions $-t \log \left|f^{\prime}\right|$. Notice that each generalized polynomial-like mapping with one critical point was proved in [7] to be quasiconformally conjugate to a polynomial. The case of a larger number of critical points can be treated similarly. Since such a conjugacy is Hölder continuous, it might turn out to be helpful in some parts of this section if one wants to deal with maps without parabolic points only. Since this conjugacy is usually not Lipschitz continuous, it is rather useless if parabolic points are present. In order to define the generalized polynomial-like mappings let $U \subset \mathbb{C}$ be an open Jordan domain with smooth boundary and let $\left\{U_{j}\right\}_{j=1}^{l}$ be a finite family of Jordan domains contained in $U$ and with mutually disjoint closures. A map

$$
f: \bigcup_{j=1}^{l} U_{j} \rightarrow U
$$

is called a generalized polynomial-like mapping (GPL) if $f$ extends holomorphically to an open neighborhood of $\bigcup_{j=1}^{l} U_{j}$ and for all $j=1, \ldots, l$, the restriction $\left.f\right|_{U_{j}}: U_{j} \rightarrow U$ is a surjective branched covering map. The branched points of $f$ coincide of course with Crit $(f)$, the set of all critical points of $f$, and we denote by $\operatorname{Br}$ the set of $i \in\{1, \ldots, l\}$ such that $U_{i}$ contains a critical point of $f$. We call $\mathrm{Br}$ the set of branched indices. If $j \in \mathrm{Br}$, then we denote by $C_{j}$ the set of critical points of $f$ contained in $U_{j}$. We also assume that if $\partial U_{j} \cap \partial U \neq \emptyset$ for some $j \in\{1, \ldots, l\}$, then this intersection is a singleton consisting of a periodic parabolic point. Following tradition we call the branched conformal GPL $f$ critically non-recurrent if for all $j \in \mathrm{Br}$,

$$
\bar{U}_{j} \cap \bigcup_{n \geq 1} f^{n}\left(C_{j}\right)=\emptyset .
$$

We finally call a GPL critically tame if there exists $1 \leq j \leq l$ such that

$$
U_{j} \cap \bigcup_{n \geq 1} f^{n}(\operatorname{Crit}(f))=\emptyset, \quad \bar{U}_{j} \subset U, \quad U_{j} \cap J(f) \neq \emptyset,
$$


where $J(f)$ is the Julia set of $f$, i.e. the boundary of the set of those points in $U$ whose forward iterates under $f$ are all well defined. In the context of critically tame GPLs we will always assume without loss of generality that the distinguished index $j$ is equal to 1 . Notice that each critically non-recurrent GPL with one critical point is critically tame if for the branched index $j$, $\bar{U}_{j} \subset U$. There are of course critically tame GPLs which are not recurrent. It can be easily verified that everything proven so far for rational critically non-recurrent functions can also be proven for critically non-recurrent GPLs. Let $g: \bar{U} \rightarrow \mathbb{R}$ be a Hölder continuous function such that

$$
\mathrm{P}(g)=\mathrm{P}\left(g,\left.f\right|_{J(f)}\right)>\sup \left\{\left.g\right|_{J(f)}\right\} .
$$

Similarly to [3] but more easily, due to the fact that the disk $U$ is already given, we can prove the following.

THEOREM 5.1. Let $f$ be a generalized polynomial-like mapping and let $g: \bar{U} \rightarrow \mathbb{R}$ be a Hölder continuous function such that $\mathrm{P}(g)=\mathrm{P}\left(g,\left.f\right|_{J(f)}\right)>$ $\sup \left\{\left.g\right|_{J(f)}\right\}$. Then

(a) There exists a unique Borel probability measure $m$ supported on $J(f)$ such that

$$
m(f(A))=\int_{A} e^{\mathrm{P}(g)-g} d m
$$

for every Borel set $A \subset \bigcup_{j=1}^{l} U_{j}$ such that $\left.f\right|_{A}$ is 1-to-1. The measure $m$ is atomless.

(b) There exists a unique Borel probability $f$-invariant measure $\mu$ absolutely continuous with respect to $m$. In addition, $\mu$ is ergodic and the Radon-Nikodym derivative $\psi=d \mu / d m: J(f) \rightarrow[0, \infty)$ has in $L^{1}(\mu)$ a continuous version which is bounded away from zero.

We will need the following technical mixing type result.

Lemma 5.2. Assume the same as in Theorem 5.1. Then there exists $0<$ $\eta<1$ such that

$$
\mu\left(\bigcap_{j=0}^{n-1} f^{-j}\left(U \backslash U_{1}\right)\right) \leq \eta^{n}
$$

for all $n \geq 1$.

Proof. It follows from Theorem 5.1 that

$$
0<\underline{\psi}:=\inf \{\psi(z): z \in J(f)\} \leq \bar{\psi}:=\sup \{\psi(z): z \in J(f)\}<\infty .
$$

Consider a partition $D_{1}, \ldots, D_{q}$ (modulo a set of $\mu$-measure zero) of $U$ such that all holomorphic inverse branches of $f$ are well defined on each $D_{j}$, $j=1, \ldots, q$. Such a partition exists since, due to Theorem 5.1, the measures $m$ and $\mu$ are atomless (in particular $m(f(\operatorname{Crit}(f)))=\mu(f(\operatorname{Crit}(f)))=0)$. Notice also that due to the same theorem the map $f$ is non-singular with 
respect to both $m$ and $\mu$. For every $i \in\{1, \ldots, q\}$ choose one holomorphic inverse branch $f_{i}^{-1}$ of $f$ mapping $D_{i}$ into $U_{1}$. Applying Theorem 5.1 we get, for every Borel set $A \subset U$,

$$
\begin{aligned}
\mu\left(U_{1} \cap f^{-1}(A)\right) & \geq \mu\left(\bigcup_{i=1}^{q} f_{i}^{-1}\left(A \cap D_{i}\right)\right)=\sum_{i=1}^{q} \mu\left(f_{i}^{-1}\left(A \cap D_{i}\right)\right) \\
& \geq \underline{\psi} \sum_{i=1}^{q} m\left(f_{i}^{-1}\left(A \cap D_{i}\right)\right)=\underline{\psi} \sum_{i=1}^{q} \int_{A \cap D_{i}} e^{g \circ f_{i}^{-1}-\mathrm{P}(g)} d m \\
& \geq \underline{\psi} e^{-\mathrm{P}(g)} e^{-\|g\|_{\infty}} \sum_{i=1}^{q} m\left(A \cap D_{i}\right)=\underline{\psi} e^{-\left(\mathrm{P}(g)+\|g\|_{\infty}\right)} m(A) \\
& =\gamma \mu(A),
\end{aligned}
$$

where $\gamma=\underline{\psi} \bar{\psi}^{-1} e^{-\left(\mathrm{P}(g)+\|g\|_{\infty}\right)}>0$. Therefore

$$
\begin{aligned}
\mu\left(\left(U \backslash U_{1}\right) \cap f^{-1}(A)\right) & =\mu\left(f^{-1}(A)\right)-\mu\left(U_{1} \cap f^{-1}(A)\right) \\
& =\mu(A)-\mu\left(U_{1} \cap f^{-1}(A)\right) \\
& \leq \mu(A)-\gamma \mu(A)=(1-\gamma) \mu(A) .
\end{aligned}
$$

Thus

$$
\begin{aligned}
\mu\left(\bigcap_{j=0}^{n-1} f^{-j}\left(U \backslash U_{1}\right)\right) & =\mu\left(\left(U \backslash U_{1}\right) \cap f^{-1}\left(\bigcap_{j=0}^{n-2} f^{-j}\left(U \backslash U_{1}\right)\right)\right) \\
& \leq(1-\gamma) \mu\left(\bigcap_{j=0}^{n-2} f^{-j}\left(U \backslash U_{1}\right)\right)
\end{aligned}
$$

and the lemma follows by induction upon taking $\eta=1-\gamma$.

From now on we assume that

$$
f: \bigcup_{j=1}^{l} U_{j} \rightarrow U
$$

is a critically tame GPL. Our first aim is to associate with $f$ a conformal infinite (hyperbolic) iterated function system satisfying all the requirements from [9]. Since $f$ is critically tame, there exists an open topological disk $V \supset \bar{U}_{1}$ whose closure is contained in $U$ and is disjoint from $\bar{U}_{2} \cup \ldots \cup \bar{U}_{l}$. In particular

For every $n \geq 1$ let

$$
V \cap \bigcup_{n \geq 1} f^{n}(\operatorname{Crit}(f))=\emptyset
$$

$$
R_{n}=\left\{z \in U_{1}: f^{n}(z) \in U_{1} \text { and } U_{1} \cap\left\{f^{k}(z): k=1, \ldots, n-1\right\}=\emptyset\right\} .
$$

That is, $R_{n}$ is the set of those points in $U_{1}$ whose first return time to $U_{1}$ is $n$. Let now $x \in R_{n}$. In view of (5.1) there exists a unique holomorphic inverse 
branch $f_{x}^{-n}: V \rightarrow \overline{\mathbb{C}}$ of $f^{n}$ sending $f^{n}(x)$ to $x$. Notice that $f_{x}^{-n}(V) \subset$ $U_{1} \subset V$. Since if $y$ is another point in $R_{n}$, then either $f_{y}^{-n}=f_{x}^{-n}$ or $f_{x}^{-n}(V) \cap f_{x}^{-n}(V)=\emptyset$, we conclude that there exists a finite set $A_{n} \subset R_{n}$ such that $f_{x}^{-n}(V) \cap f_{y}^{-n}(V)=\emptyset$ for all $x, y \in A_{n}, x \neq y$, and for every $z \in R_{n}$ there exists $w \in A_{n}$ such that $f_{z}^{-n}=f_{w}^{-n}$. Therefore, the countable family

$$
S=\left\{f_{x}^{-n}: V \rightarrow V, f_{x}^{-n}: \bar{U}_{1} \rightarrow \bar{U}_{1}\right\}_{n \geq 1, x \in A_{n}}
$$

forms a conformal iterated function system in the sense of [9]. We will frequently denote the elements of $S$ by $\phi_{i}, i \in I$. Given $\omega=\omega_{1} \ldots \omega_{n} \in I^{n}$, we put

$$
\phi_{\omega}=\phi_{\omega_{1}} \circ \ldots \circ \phi_{\omega_{n}} .
$$

Let $N(i)$ denote the only integer satisfying $\phi_{i}=f_{x}^{-N_{i}}$ for some $x \in A_{N_{i}}$. Given $t \geq 0$ and $s \in \mathbb{R}$ we introduce the family $G_{t, s}=\left\{g_{i, t, s}: V \rightarrow \mathbb{R}\right\}_{i \in I}$ by the formulas

$$
g_{i, t, s}(x)=t \sum_{j=1}^{N_{i}} g \circ f^{j}\left(\phi_{i}(x)\right)-s N(i) .
$$

Recall that $p=\max \{p(\omega): \omega \in \Omega\}$. Let us prove the following.

LEMMA 5.3. Suppose that $f$ is a critically tame and critically non-recurrent GPL. If $f$ has no parabolic points, then all the functions $g_{i, t, s}, i \in I$, are Hölder continuous with the same Hölder exponent and the same Hölder constant. If $f$ has parabolic points, then the same is true assuming additionally that the Hölder exponent of $g$ is greater than $p /(p+1)$.

Proof. Notice first that $U_{1}$ has no parabolic points. In the case of the lack of parabolic points, this immediately follows from Lemma 7.7 of [18] (the stars can be dropped there); cf. [1]. So, suppose that $\Omega \neq \emptyset$. It then follows from (5.1) and Remark 4.4 that $\operatorname{diam}\left(V_{n}\right) \leq B n^{-(p+1) / p}$ for all $n \geq 1$ and all connected components $V_{n}$ of $f^{-n}(V)$. Hence, it follows from Koebe's distortion theorem that $\left|\left(f_{*}^{-n}\right)^{\prime}(x)\right| \leq \widetilde{B}(n+1)^{-(p+1) / p}$ for all $n \geq 0$, all holomorphic inverse branches $f_{*}^{-n}: V \rightarrow U$ of $f^{n}$, all $x \in \bar{U}_{1}$ and some $\widetilde{B}>0$. In particular if $y, z \in \bar{U}_{1}$, then for all $i \in I$,

$$
\begin{aligned}
\left|\sum_{j=1}^{N_{i}} g \circ f^{j}\left(\phi_{i}(z)\right)-\sum_{j=1}^{N_{i}} g \circ f^{j}\left(\phi_{i}(y)\right)\right| \leq \sum_{j=1}^{N_{i}}\left|g \circ f^{j}\left(\phi_{i}(z)\right)-g \circ f^{j}\left(\phi_{i}(y)\right)\right| \\
\quad \leq \sum_{j=1}^{N_{i}} C\left|f^{j}\left(\phi_{i}(z)\right)-f^{j}\left(\phi_{i}(y)\right)\right|^{\alpha} \\
\quad \leq \sum_{j=1}^{N_{i}} C \widetilde{B}\left(N_{i}+1-j\right)^{-(p+1) / p}|z-y|^{\alpha} \leq \sum_{j=1}^{\infty} k^{-\alpha(p+1) / p}|z-y|^{\alpha},
\end{aligned}
$$

where $\alpha$ is the Hölder exponent of $g$ and $C$ is the Hölder constant. 
Lemma 5.4. For all $t \geq 0$ and $s \in \mathbb{R}, G_{t, s}$ is a Hölder family of functions in the sense of [5] and [10].

Proof. By Montel's theorem the family $\left\{\phi_{i}: V \rightarrow U_{1}\right\}_{i \in I}$ is normal, and since $U_{1} \cap J(f) \neq \emptyset$, all its limit functions are constant. Therefore all the limit functions of the derivatives $\phi_{i}^{\prime}$ of $\phi_{i}$ are equal to the constant function 0 . Thus, there exists $q \geq 1$ such that $\left\|\phi_{\omega}^{\prime}\right\|_{\infty} \leq 1 / 2$ for all $\omega \in I^{n}$ with $n \geq q$, where $\|\cdot\|_{\infty}$ is the supremum norm on $\bar{U}_{1}$. Notice also that by Koebe's distortion theorem (and since $\bar{U}_{1} \subset V$ ),

$$
Q=\sup \left\{\left\|\phi_{\omega}^{\prime}\right\|_{\infty}: \omega \in I^{n}, 1 \leq n \leq q-1\right\}<\infty .
$$

Fix now $n \geq 1, \omega \in I^{n}$, and two points $x, y \in \bar{U}_{1}$. Write $n-1=k q+r$, where $0 \leq r \leq q-1$. Then $\left\|\phi_{\omega}^{\prime}\right\|_{\infty} \leq(1 / 2)^{k} Q \leq Q(1 / 2)^{(n-1) / q}$ and therefore

$$
\operatorname{diam}\left(\phi_{\omega}\left(\bar{U}_{1}\right)\right) \leq \bar{Q}\left(2^{-1 / q}\right)^{n-1}
$$

where $\bar{Q}$ is the constant depending on $Q$, the diameter of $U_{1}$, and the maximal number of segments needed to join each point in $\bar{U}_{1}$ with an arbitrarily frozen point in $U_{1}$ (note that $U_{1}$ is not assumed to be convex nor of the star shape). Using Lemma 5.3, with the Hölder exponent $\alpha$ following from Lemma 5.3, we conclude that

$$
\begin{aligned}
\left|g_{\omega_{1}, t, s}\left(\phi_{\sigma(\omega)}(y)\right)-g_{\omega_{1}, t, s}\left(\phi_{\sigma(\omega)}(x)\right)\right| & \leq C_{1}\left|\phi_{\sigma(\omega)}(y)-\phi_{\sigma(\omega)}(x)\right|^{\alpha} \\
& \leq C_{1} \bar{Q}\left(2^{-\alpha / q}\right)^{n-1}
\end{aligned}
$$

for some universal constant $C_{1}$ depending on $t$.

We recall (see [10]; cf. [5], where a different terminology was used) that a Hölder family $\left\{q_{i}: \bar{U}_{1} \rightarrow \mathbb{R}\right\}_{i \in I}$ is called summable if

$$
\sum_{i \in I} e^{\sup \left(q_{i}\right)}<\infty
$$

Using Lemma 5.2 let us prove the following.

LEMma 5.5. Assume the same as in Lemma 5.3. If $\mathrm{P}(g)>\sup (g)$, then there exists $\delta>0$ such that if $(t, s) \in(1-\delta, 1+\delta) \times(\mathrm{P}(g)-\delta, \infty)$, then $G_{t, s}$ is a summable Hölder family of functions.

Proof. The fact that the families $G_{t, s}$ are Hölder has been proved in Lemma 5.4. Since

$$
R_{n}=U_{1} \cap \bigcap_{j=1}^{n-1} f^{-j}\left(U \backslash U_{1}\right) \cap f^{-n}\left(U_{1}\right) \subset f^{-1}\left(\bigcap_{j=0}^{n-2} f^{-j}\left(U \backslash U_{1}\right)\right)
$$

and since the measure $\mu$ is $f$-invariant, it follows from Lemma 5.2 that $\mu\left(R_{n}\right) \leq \eta^{n-1}$. Applying now Theorem 5.1(b), we infer that there exists a constant $C_{1}>0$ such that 


$$
m\left(R_{n}\right) \leq C_{1} \eta^{n}
$$

for all $n \geq 1$. Combining this, Lemma 5.3 and Theorem 5.1, we conclude that there exists a constant $C_{2}>0$ such that

$$
\sum_{w \in A_{n}} \exp \left(\sup _{\bar{U}_{1}}\left(\sum_{j=1}^{n} g \circ f^{j} \circ f_{w}^{-1}-\mathrm{P}(g) n\right)\right) \leq C_{2} \eta^{n}
$$

for all $n \geq 1$. Hence, for every $t \geq 0$ and all $s \in \mathbb{R}$, we get

$$
\begin{aligned}
& \sum_{w \in A_{n}} \exp \left(\sup _{\bar{U}_{1}}\left(\sum_{j=1}^{n} t g \circ f^{j} \circ f_{w}^{-1}-s n\right)\right) \\
&=\sum_{w \in A_{n}} \exp \left(\operatorname { s u p } _ { \overline { U } _ { 1 } } \left(\sum_{j=1}^{n} g \circ f^{j} \circ f_{w}^{-1}-\mathrm{P}(g) n\right.\right. \\
&\left.\left.+(t-1) \sum_{j=1}^{n} g \circ f^{j} \circ f_{w}^{-1}+(\mathrm{P}(g) n-s n)\right)\right) \\
& \leq \exp \left(|t-1|\|g\|_{\infty} n\right) \exp ((\mathrm{P}(g)-s) n) \\
& \quad \times \sum_{w \in A_{n}} \exp \left(\sup _{\bar{U}_{1}}\left(\sum_{j=1}^{n} g \circ f^{j} \circ f_{w}^{-1}-\mathrm{P}(g) n\right)\right) \\
& \leq C_{2} \exp (-\kappa n) \exp ((\mathrm{P}(g)-s) n) \exp \left(|t-1|\|g\|_{\infty} n\right),
\end{aligned}
$$

where $\kappa=-\log \eta$. Taking now $\delta=\kappa\left(4\left(1+\|g\|_{\infty}\right)^{-1}\right.$, we conclude that for all $(t, s) \in(1-\delta, 1+\delta) \times(\mathrm{P}(g)-\delta, \infty)$ and all $n \geq 1$,

$$
\sum_{w \in A_{n}} \exp \left(\sup _{\bar{U}_{1}}\left(\sum_{j=1}^{n} t g \circ f^{j} \circ f_{w}^{-1}-s n\right)\right) \leq C_{2} \exp (-\kappa n / 2)
$$

and therefore

$$
\sum_{n \geq 1} \sum_{w \in A_{n}} \exp \left(\sup _{\bar{U}_{1}}\left(\sum_{j=1}^{n} t g \circ f^{j} \circ f_{w}^{-1}-s n\right)\right)<\infty .
$$

Since both functions $g \mapsto \mathrm{P}(g)$ and $g \mapsto \sup (g)$ are continuous, we get the following.

Lemma 5.6. If $\mathrm{P}(g)>\sup (g)$, then there exists $\delta>0$ such that $\mathrm{P}(t g)>$ $\sup (t g)$ for all $t \in(1-\delta, 1+\delta)$.

Given $\omega=\omega_{1} \ldots \omega_{n} \in I^{n}, n \geq 1$, let $\sigma(\omega)=\omega_{1} \ldots \omega_{n-1}$. Recall from [5] and [10] that the topological pressure $\mathrm{P}\left(G_{t, s}\right)$ of the family $G_{t, s}$ is defined as follows: 


$$
\begin{aligned}
\mathrm{P}\left(G_{t, s}\right) & =\lim _{n \rightarrow \infty} \frac{1}{n} \log \sum_{|\omega|=n}\left\|\exp \left(\sum_{j=1}^{n} g_{\omega_{j}, t, s} \circ \phi_{\sigma^{j} \omega}\right)\right\|_{0} \\
& =\lim _{n \rightarrow \infty} \frac{1}{n} \log \sum_{|\omega|=n} \exp \left(\sup _{\bar{U}_{1}} \sum_{j=1}^{n} g_{\omega_{j}, t, s} \circ \phi_{\sigma^{j} \omega}\right) .
\end{aligned}
$$

Consider the iterated function system

$$
S=\left\{f_{x}^{-n}: V \rightarrow V, f_{x}^{-n}: \bar{U}_{1} \rightarrow \bar{U}_{1}\right\}_{n \geq 1, x \in A_{n}}
$$

Its limit set is defined as follows:

$$
J_{S}=\bigcap_{n=1}^{\infty} \bigcup_{|\tau|=n} \phi_{\tau}\left(\bar{U}_{1}\right)
$$

For an alternative definition and further properties of $J_{S}$ see [9] and [10] for example. Let us also recall $([5],[10])$ that the $G_{t, s}$-conformal measure $m_{G_{t, s}}$ supported on $J_{S}$ is uniquely determined by the following two properties:

$$
\begin{gathered}
m_{G_{t, s}}\left(\phi_{i}(A)\right)=\int_{A} \exp \left(g_{i, t, s}-\mathrm{P}(F)\right) d m_{G_{t, s}}, \quad \forall i \in I, \\
m\left(\phi_{i}\left(\bar{U}_{1}\right) \cap \phi_{j}\left(\bar{U}_{1}\right)\right)=0 \quad \text { for all } i \neq j .
\end{gathered}
$$

Let $\delta(g)>0$ be the minimum of the numbers $\delta$ from Lemmas 5.5 and 5.6. For $t \in(1-\delta, 1+\delta)$ let $m_{t}$ be the measure produced by Theorem 5.1 (a) for the potential $t \phi$.

We now prove the following.

LEMMA 5.7. If $t \in(1-\delta(g), 1+\delta(g))$, then

$$
\mathrm{P}\left(G_{t, \mathrm{P}(t g)}\right)=0 \quad \text { and } \quad m_{G_{t, \mathrm{P}(t g)}}=\left.m_{t}\right|_{J_{S}} .
$$

Proof. Denote $\mathrm{P}\left(G_{t, \mathrm{P}(t g)}\right)$ by $\widehat{\mathrm{P}}(t)$ and $m_{G_{t, \mathrm{P}(t)}}$ by $\widehat{m}_{t}$. By the definitions of the measures $\widehat{m}_{t}, m_{t}$ and by Lemmas $5.4,5.6$ and Theorem 5.1 , for every $\tau \in I^{*}=\bigcup_{n \geq 1} I^{n}$, we have

$$
\begin{aligned}
\frac{\widehat{m}_{t}\left(\phi_{\tau}\left(U_{1}\right)\right)}{m_{t}\left(\phi_{\tau}\left(U_{1}\right)\right)} & =\frac{\int \exp \left(\sum_{j=1}^{|\tau|} g_{\tau_{j}, t, \mathrm{P}(t g)}\right) \exp (-\widehat{\mathrm{P}}(t)|\tau|) d \widehat{m}_{t}}{\int \exp \left(\sum_{j=1}^{|\tau|} g_{\tau_{j}, t, \mathrm{P}(t g)}\right) d m_{t}} \\
& \asymp \exp (-\widehat{\mathrm{P}}(t)|\tau|) .
\end{aligned}
$$

So, if $\widehat{\mathrm{P}}(t)>0$, then $\widehat{m}_{t}\left(J_{S}\right)=0$, and if $\widehat{\mathrm{P}}(t)<0$, then $m_{t}\left(J_{S}\right)=0$, which contradicts the fact that $m_{t}\left(J_{S}\right)=m_{t}\left(U_{1}\right)>0$; the latter follows by a straightforward induction from the formula $m_{t}\left(\bigcup_{i \in I} \phi_{i}\left(U_{1}\right)\right)=m_{t}\left(U_{1}\right)$ resulting from Poincaré's recurrence theorem. Thus $\widehat{\mathrm{P}}(t)=0$ and the first part of our lemma is proven. The equality $\widehat{m}_{t}=\left.m_{t}\right|_{J_{S}}$ follows now from (5.2) con- 
sidered as two formulas: one for the numerator and one for the denominator, along with either Theorem 3.1.7 of [10] or Corollary 2.12 of [5].

Following [10] and [5] we call $g_{t, s}: I^{\infty} \rightarrow \mathbb{R}$ defined by the formula

$$
g_{t, s}(\tau)=g_{\tau_{1}, t, s}(\pi(\sigma \tau))
$$

the amalgamated function of the family $G_{t, s}$. Here $\pi: I^{\infty} \rightarrow \mathbb{C}$ is the projection associated with the conformal iterated function system $S$ and $\sigma: I^{\infty} \rightarrow I^{\infty}$ is the shift map. We are now in a position to prove the following.

THEOREM 5.8. Assume that $f$ is a critically tame and critically nonrecurrent GPL, and that $\phi$ is Hölder continuous, with exponent greater than $p /(p+1)$ if $f$ has parabolic points. Then the function $t \mapsto \mathrm{P}(t g), t \in(1-\delta$, $1+\delta)$, is real-analytic.

Proof. Let $\widehat{m}_{t}=m_{G_{t, \mathrm{P}(t g)}}$. In view of Theorem 2.6.12 of [10] (see also [19] and [5]) and Lemma 5.5, the function

$$
(t, s) \mapsto \mathrm{P}\left(g_{t, s}\right)=\mathrm{P}\left(G_{t, s}\right), \quad(t, s) \in(1-\delta, 1+\delta) \times(\mathrm{P}(g)-\delta, \mathrm{P}(g)+\delta),
$$

is real-analytic. In view of Lemma 5.7 and the implicit function theorem, it is therefore now enough to demonstrate that $\frac{\partial \mathrm{P}}{\partial s}(t, s) \neq 0$ at the point $(t, \mathrm{P}(t g))$ for every $t \in(1-\delta, 1+\delta)$. Indeed, let $\widetilde{\sim}_{t}, t \in(1-\delta, 1+\delta)$, be the shift invariant measure on $I^{\infty}$ equivalent to $\widetilde{\widehat{m}}_{t}$, the lift of $\widehat{m}_{t}$ to the coding space $I^{\infty}$. Since $\mu_{t}$, the measure appearing in Theorem 5.1(b) for the potential $t g$, is $f$-invariant and the system $S$ is defined according to the first-return time, $\mu_{t}$ is $S$-invariant in the sense that

$$
\mu_{t}\left(\bigcup_{i \in I} \phi_{i}(A)\right)=\sum_{i \in I} \mu_{t}\left(\phi_{i}(A)\right)=\mu_{t}(A)
$$

for every Borel set $A \subset J_{S}$. Hence, using the last part of Lemma 5.7, we deduce that $\left.\mu_{t}\right|_{J_{S}}=\widetilde{\mu}_{t} \circ \pi^{-1}$. Therefore applying Proposition 2.6.13 of [10] (see also [19] and [5]) along with Kac's lemma, we obtain

$$
\frac{\partial \mathrm{P}}{\partial s}(t, \mathrm{P}(t g))=-\int_{I^{\infty}} N\left(\tau_{1}\right) d \widetilde{\mu}_{t}=-\left.\int_{J_{S}} N d \mu_{t}\right|_{J_{S}}=-\frac{1}{\mu_{t}\left(J_{S}\right)} \neq 0,
$$

where after the second equality sign, we treated (slightly informally) the function $N$ as defined on $J_{S}$.

After this paper has been written the analogous result for potentials of the form $-t \log \left|f^{\prime}\right|$ was established in [15].

Acknowledgments. The author wishes to thank the referee and Feliks Przytycki whose comments and suggestions improved the final version of this paper. 


\section{References}

[1] L. Carleson, P. Jones and J.-C. Yoccoz, Julia and John, Bol. Soc. Brasil. Mat. 25 (1994), 1-30.

[2] M. Denker and M. Urbański, On the existence of conformal measures, Trans. Amer. Math. Soc. 328 (1991), 563-587.

[3] - - - Ergodic theory of equilibrium states for rational maps, Nonlinearity 4 (1991), 103-134.

[4] - - - On Sullivan's conformal measures for rational maps of the Riemann sphere, ibid. 4 (1991), 365-384.

[5] P. Hanus, D. Mauldin and M. Urbański, Thermodynamic formalism and multifractal analysis of conformal infinite iterated function systems, Acta Math. Hungar. 96 (2002), 27-98.

[6] N. Haydn and S. Isola, Parabolic rational maps, preprint, 1999.

[7] G. Levin and S. van Strien, Local connectivity of the Julia set of real polynomials, Ann. of Math. 147 (1998), 471-541.

[8] M. Martens, The existence of $\sigma$-finite invariant measures, Applications to real onedimensional dynamics, preprint.

[9] D. Mauldin and M. Urbański, Dimensions and measures in infinite iterated function systems, Proc. London Math. Soc. (3) 73 (1996) 105-154.

[10] - - - Graph directed Markov systems, to appear, available on the author's webpage.

[11] C. McMullen, Complex Dynamics and Renormalization, Ann. of Math. Stud. 135, Princeton Univ. Press, 1994.

[12] F. Przytycki, Conical limit set and Poincaré exponent for iterations of rational functions, Trans. Amer. Math. Soc. 351 (1999), 2081-2099.

[13] F. Przytycki, J. Rivera-Letelier and S. Smirnov, Equivalence and topological invariance of conditions for non-uniform hyperbolicity in the iteration of rational maps, Invent. Math. 151 (2003), 29-63.

[14] F. Przytycki and M. Urbański, Fractals in the plane-ergodic theory methods, to appear.

[15] B. Stratmann and M. Urbański, Real analyticity of topological pressure for parabolically semi-hyperbolic generalized polynomial-like maps, Indag. Math., to appear.

[16] -, -, Multifractal analysis of parabolically semi-hyperbolic generalized polynomiallike maps, in preparation.

[17] M. Urbański, Rational functions with no recurrent critical points, Ergodic Theory Dynam. Systems 14 (1994), 391-414.

[18] - Geometry and ergodic theory of conformal nonrecurrent dynamics, ibid. 17 (1997), 1449-1476.

[19] M. Urbański and Z. Zinsmeister, Geometry of hyperbolic Julia-Lavaurs sets, Indag. Math. 12 (2001), 273-292.

Department of Mathematics

University of North Texas

P.O. Box 311430

Denton, TX 76203-1430, U.S.A.

E-mail: urbanski@unt.edu

Web: http://www.math.unt.edu/ ${ }^{\sim}$ urbanski 